\title{
Quantitative Normal Force Measurements by Means of Atomic Force Microscopy Towards the Accurate and Easy Spring Constant Determination
}

\author{
Andrzej Sikora \\ Electrotechnical Institute, Division of Electrotechnology and Materials Science, M. Skłodowskiej-Curie Wrocław, Poland \\ Email address: \\ sikora@iel.wroc.pl \\ To cite this article: \\ Andrzej Sikora. Quantitative Normal Force Measurements by Means of Atomic Force Microscopy Towards the Accurate and Easy Spring \\ Constant Determination. Nanoscience and Nanometrology. Vol. 2, No. 1, 2016, pp. 8-29. doi: 10.11648/j.nsnm.20160201.12
}

\begin{abstract}
Due to its rapid popularity increase within last three decades, with particular focus on submicrometer quantitative surface's properties imaging, atomic force microscopy (AFM) is still a subject of development and research in terms of both better understanding and efficient utilization of various measurement techniques. Quantitative and comparable measurements at nanoscale are a significant issue, as both: science and industry desire reliable results, allowing to perform repetitive experiments at any time and location. Therefore a numerous analysis and research projects were carried out to provide metrological approach for those techniques in terms of providing the traceability and the uncertainty estimation. In this paper an overview of various methods and approaches towards quantitative determination of the normal spring constant of the AFM probes is presented.
\end{abstract}

Keywords: Atomic Force Microscopy, Scanning Probe, Spring Constant, Calibration, Normal Force Measurement, Force Spectroscopy

\section{Introduction}

Since its development, [1] atomic force microscopy became very popular measurement method, providing a wide range of micron and submicron - scale diagnostics of the surface. The application range of various AFM techniques very quickly covered wide range of science and technology, as the diversity of the information can be obtained [2-4]. Taking into account the sample preparation simplicity and possibility of performing the measurements in wide range of the conditions such as vacuum [5], air [6], various gases [7] and liquids [8] a rapid growth of research using AFM was observed. As the consequence, this progress caused traceability demand, as one desires a quantitative approach allowing to compare the results acquired using various instruments, by different research groups or industrial entities. A number of attempts were performed in order to develop a suitable instrumental and procedural methodology allowing to calibrate the measurement setups and establish the description of specific properties of nanoworld basing on SI units system. As different properties of the surface can be measured with AFM, the variety of the approaches was observed regarding to specific measuring techniques: scanning thermal microscopy in terms of measuring local temperature as well as the thermal conductivity of the surface $[9,10]$, scanning capacitance microscopy [11], conductive probe AFM [12], magnetic force microscopy [13], electrostatic force microscopy [14], Digital pulsed force imaging [15], Kelvin probe force microscopy [16].

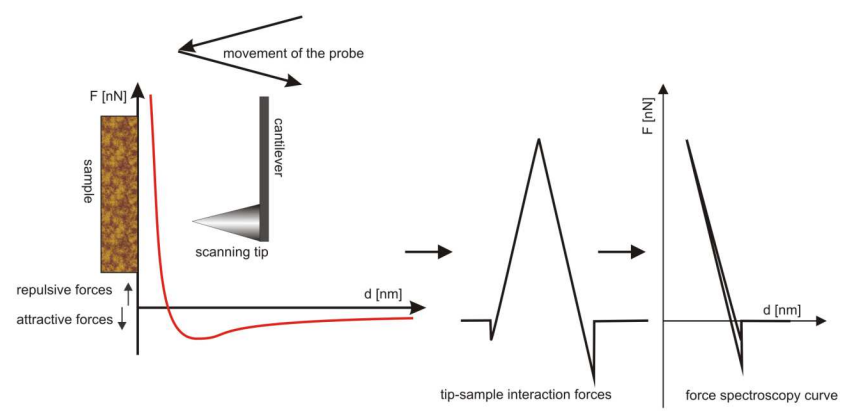

Fig. 1. The force spectroscopy measurement procedure in terms of the tip-sample distance changes and specific interaction forces acquisition.

So far however, as one of the first AFM measurement modes, the force spectroscopy is commonly applied in quantitative fashion, as a number of works provided reliable calibration solutions. This technique bases on the observation of the probes response on the tip-sample interaction while the distance between the tip and surface decreases until contact is 
obtained, and increases in order to retract the tip to the distance where no any interaction with the surface is observed [17]. Figure 1 illustrates the force spectroscopy measurement process and its simplified outcome (force curve in time domain and force-distance curve). One has to be aware of the simplifications of presented interaction diagram and forces curves, as only general impression of the method is shown.

It should be underlined, that due to selective tip-molecule contact, one can obtain a unique information about investigated object, as no other method is able to provide such an outcome [18]. One has to be aware, that due to the complexity of the tip-sample interactions, the specific results of the measurement may vary in terms of the experiment outcome, the tip-sample distance control and the interpretation. Some of the examples of practical utilization of the force spectroscopy covering various scientific areas are listed below:

- physics: the investigation of the physical principles of the adhesion changes due to varying the tip-sample distance changes speed [19], the measurements of the physical bond of the fullerene [20],

- biology: the measurements of the mechanical properties of the cells and tissue [21] or the proteins unfolding observation [22],

- chemistry: the investigation of polyazomethine compound provided an insight in the mechanical interaction between the scanning tip and submicron features, revealing their wear while the pressing cycles are repeated [23], the observation of the interaction between two poly(benzyl ether) dendrons in terms of observation of the rupture force, which indicated thermodynamic pulling property [24],

- material science: the deterioration of the material's surfaces due to the environmental conditions may be observed and quantified with much higher sensitivity than in case of traditional methods defined by standards [25],

- forensics: distinguishing of various types of adhesive tapes by the measurement of the adhesion force and adhesion energy [26], the determination of the blood spots age by the determination of the Young modulus [27],

- nanotribology: the investigation of various materials in terms of the resistance for mechanical wear [28].

Also unique applications of the AFM probes can be found. Serrel et al. developed the microfabricated setup allowing to measure the force applied uniaxially to an adherent fibroblast until de-adhering of the cell was observed [29]. The micromechanical force detector was calibrated using AFM probe, with determined $8 \%$ spring constant error. It allowed to obtain approximately $10 \%$ of the standard uncertainty of complete setup.

It should be underlined, that in abovementioned examples, the range of the measured forces is wide. Therefore one has an insight into the complexity of the calibration issue. In many cases the developed measurement technique is useful only when the probes revealing specific mechanical properties are used. In particular, the spring constant of probes is an essential factor, as it determines the response of the cantilever to a certain force. Although the spring constant of commercially available probes can be easily found in the product specification, the range that this factor can vary, may reach even two orders of magnitude (tab. 1), therefore the AFM operator has to perform the calibration procedure. It should be underlined, that one can also purchase probes, where due to the technological process and the construction design, much smaller variations of the spring constant are declared [30]. This is, however, a small fraction of the market.

Table 1. Short comparison of the typical parameters of the several AFM probes, including the spring constant and resonance frequency ranges.

\begin{tabular}{|c|c|c|c|c|c|c|c|}
\hline producer & model & mode & material & $\begin{array}{l}\text { Dimensions } \\
\text { l/w/t [ } \quad \mathrm{m}]\end{array}$ & $\begin{array}{l}\mathrm{k}[\mathrm{N} / \mathrm{m}] \\
\mathrm{min} / \mathrm{typ} . / \mathrm{max}\end{array}$ & $\begin{array}{l}f_{\text {rez }}[\mathrm{kHz}] \\
\mathrm{min} / \mathrm{typ} . / \mathrm{max}\end{array}$ & $\mathbf{r}_{\text {tip }}[\mathbf{n m}]$ \\
\hline Bruker & RTESPA & $\mathrm{SC}^{1)}$ & $\mathrm{Si}(\mathrm{Sb}) 0.01-0.025 \Omega \mathrm{cm}$ & $125 / 35 / 3.75$ & $20 / 40 / 80$ & $200 / 300 / 400$ & 8 \\
\hline Bruker & PR-CO10 & $\mathrm{C}$ & $\mathrm{Si}(\mathrm{Sb}) 0.01-0.025 \Omega \mathrm{cm}$ & $\begin{array}{l}450 / 50 / 2 \\
120 / 25 / 0.6^{5)}\end{array}$ & $\begin{array}{l}0.02-0.2-0.77 \\
0.175 / 0.35 / 0.7\end{array}$ & $\begin{array}{l}6 / 13 / 21 \\
50 / 65 / 80\end{array}$ & 7 \\
\hline Bruker & $\mathrm{NP}-\mathrm{O} 10^{2)}$ & $\mathrm{C}$ & $\mathrm{Si}_{3} \mathrm{~N}_{4}$ & $\begin{array}{l}205 / 40 / 0.6^{5)} \\
120 / 20 / 0.6^{5)} \\
205 / 25 / 0.6^{5)}\end{array}$ & $\begin{array}{l}0.06 / 0.12 / 0.24 \\
0.12 / 0.24 / 0.48 \\
0.03 / 0.06 / 0.12\end{array}$ & $\begin{array}{l}16 / 23 / 28 \\
40 / 56 / 75 \\
12 / 18 / 24\end{array}$ & 20 \\
\hline NT-MDT & NSG01 & $\mathrm{SC}$ & $\mathrm{Si}(\mathrm{Sb}) 0.025 \Omega \mathrm{cm}$ & $125 / 30 / 2$ & $1.45 / 5.1 / 15.1$ & $87 / 150 / 230$ & 10 \\
\hline NT-MDT & CSG01 & $\mathrm{C}$ & $\mathrm{Si}(\mathrm{Sb})$ & $350 / 30 / 1.0$ & $0.003 / 0.03 / 0.13$ & $4 / 9.8 / 17$ & 6 \\
\hline MicroMasch & NSC15 & $\mathrm{SC}$ & $\mathrm{Si}(\mathrm{P})$ 0.01..0.05 $\Omega \mathrm{cm}$ & $\begin{array}{l}125 / 35 / 4 \\
250 / 35 / 2\end{array}$ & $\begin{array}{l}20 / 40 / 80 \\
0.3 / 0.8 / 2\end{array}$ & $\begin{array}{l}265 / 325 / 410 \\
30 / 40 / 55\end{array}$ & 10 \\
\hline MicroMasch & $\mathrm{CSC} 37$ & $\mathrm{C}$ & $\mathrm{Si}(\mathrm{N})$ 0.01..0.025 $\Omega \mathrm{cm}$ & $\begin{array}{l}350 / 35 / 2 \\
300 / 35 / 2\end{array}$ & $\begin{array}{l}0.1 / 0.3 / 0.6 \\
0.1 / 0.4 / 1\end{array}$ & $\begin{array}{l}15 / 20 / 30 \\
20 / 30 / 40\end{array}$ & 8 \\
\hline NanoWorld & NCST & $\mathrm{SC}$ & $\mathrm{Si}$ & $150 / 27 / 2.8$ & $3 / 7.4 / 16$ & $120 / 160 / 205$ & 8 \\
\hline NanoWorld & CONTSC & $\mathrm{C}$ & $\mathrm{Si}$ & $225 / 48 / 1.0$ & $0.02 / 0.2 / 0.7$ & $0.2 / 23 / 39$ & 8 \\
\hline Nanosensors & CNT-NCH & $\mathrm{SC}$ & $\mathrm{Si}$ & $125 / 30 / 4.0$ & $10 / 42 / 130$ & $204 / 330 / 497$ & $1.2^{3)}$ \\
\hline Nanosensors & PPP-CONT & $\mathrm{C}$ & $\mathrm{Si}$ & $450 / 50 / 2$ & $0.02 / 0.2 / 0.77$ & $6 / 13 / 21$ & 7 \\
\hline Bruker & FASTSCAN-A ${ }^{4)}$ & $\mathrm{SC}$ & $\mathrm{Si}_{3} \mathrm{~N}_{4}$ & $27 / 33 / 0.6^{5)}$ & $10 / 18 / 25$ & $800 / 1400 / 2000$ & 5 \\
\hline Olympus & OMCL-AC55TS ${ }^{4)}$ & $\mathrm{SC}$ & $\mathrm{Si}(\mathrm{n})$ & $55 / 31 / 2.35^{5)}$ & $38 / 85 / 184$ & $850 / 1600 / 2500$ & 7 \\
\hline
\end{tabular}

${ }^{1}$ contact mode $(\mathrm{C})$ semicontact/ noncontact mode (SC)

${ }^{2} 4$ probes integrated with one chip

${ }^{3}$ the carbon nanotube is used as the scanning tip (SNT 1, $2 \mathrm{~nm} /$ DNT 2,4 nm)

${ }^{4}$ the probes designed for the fast scanning AFM's (high resonance frequency)

${ }^{5} \mathrm{~V}$-shape probe 
Complete calibration of the AFM system in terms of quantitative force measurement contains two major steps: the determination of the probe's bending detection sensitivity and establishing the spring constant of the cantilever. The first parameter is relatively easy to find, as the changes of the output electrical signal is acquired while the cantilever tip is pushed against a hard surface. The spring constant determination, on the other hand, is the process, where a number of issues can have an influence on the final result. Therefore, a diversity of the approaches was developed and described in papers within last three decades, as one desires simple, robust quick and high accuracy calibration technique.

\section{The Principles of the Force Measurement Using AFM Technique}

The AFM setup contains three main elements allowing to measure the force: the probe, the probe's deflection detection system and signal processing hardware/ software unit, including precision sample positioning setup. Each of the components should be considered in terms of the final outcome of the measurement procedure if mechanical properties of the surface in general or the tip-sample interaction force in particular are considered. In following sections the principle information concerning main parts of the measurement system is provided.

\subsection{The Properties of the Scanning Probes}

In general, the probes are made of the silicon or silicon nitride. The spring constant variety in range from 0.001 to 100 $\mathrm{N} / \mathrm{m}$ is provided as the thickness, length and width of the cantilevers varies. If necessary, also very stiff, tuning fork-based probes, up to $2000 \mathrm{~N} / \mathrm{m}$ can be utilized [31, 32]. Moreover, one can purchase custom-made special probes made of sapphire with $10000 \mathrm{~N} / \mathrm{m}$ spring constant [33]. Before the experiment, the certain type of the probe with specific spring constant should be selected in order to provide the best possible surface imaging conditions as well as other properties investigation. Also the covering of the probe with specific materials may be essential, if complex measurements are planned, such as requiring functionalized tips. Figure 2 shows the essential dimensional parameters of typical AFM probe.

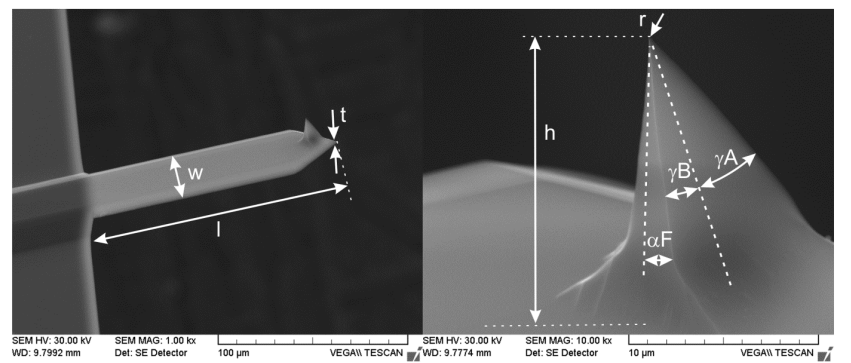

Fig. 2. The SEM view of the AFM probe with the most important dimensions marked where: $l$-length of the cantilever, $t$ - thickness of the cantilever, $w-$ width of the cantilever, $r$-radius of the tip's apex, $\gamma A, \gamma B, \gamma F$-front, back and side angles of the tip respectively, $h$-height of the tip.
According to the Hook's law, the probe's bending is proportional to the force acting on the tip. Therefore, a local interaction of the tip with the surface may be easily calculated in a quantitative fashion, using equation 1:

$$
F=k \times \Delta z
$$

where $\mathrm{F}$ is the force, $\mathrm{k}$ is the spring constant and $\Delta \mathrm{z}$ is the displacement of the probe form the 0 force distance (attractive and repulsive forces equilibrium). This relation is shown in figure 3 .

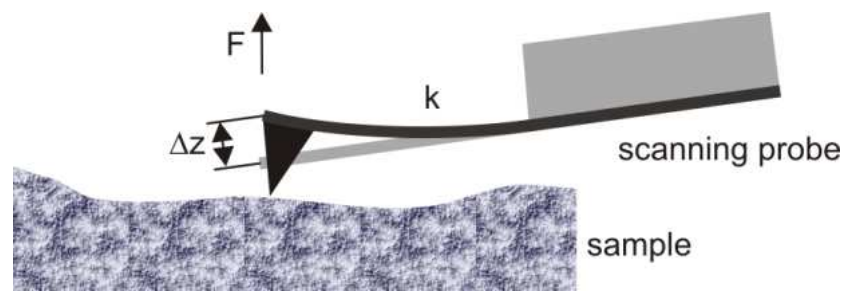

Fig. 3. The relation between the force acting on the probe and its bending.

\subsection{The Probe's Deflection Detection Systems}

Despite the variety of developed solutions allowing to detect the deflection of the probe, the most popular is the optical setup known as OBD (Optical Beam Deflection), basing on the measurement of the electrical signals ratio change from the four-section photodiode, while the cantilever is illuminated with a laser beam, which is reflected to the photodiode (fig. 4) [34].

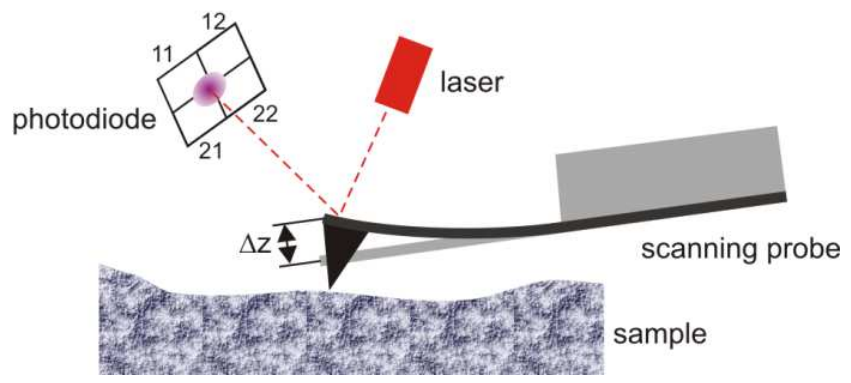

Fig. 4. The idea of the probe's deflection detection using optical beam deflection setup.

The current values for each part of the photodiode can be calculated from following formulas:

$$
\begin{aligned}
& i_{11}=\alpha \eta P \frac{\left(\frac{1}{2} a-\Delta x\right)\left(\frac{1}{2} b+\Delta y\right)}{a b} \\
& i_{12}=\alpha \eta P \frac{\left(\frac{1}{2} a+\Delta x\right)\left(\frac{1}{2} b+\Delta y\right)}{a b} \\
& i_{21}=\alpha \eta P \frac{\left(\frac{1}{2} a-\Delta x\right)\left(\frac{1}{2} b-\Delta y\right)}{a b} \\
& i_{22}=\alpha \eta P \frac{\left(\frac{1}{2} a+\Delta x\right)\left(\frac{1}{2} b-\Delta y\right)}{a b}
\end{aligned}
$$

where: $\alpha-$ is optical setup efficiency factor, $\eta$ - quantum efficiency of the detector, $P$ - total power of the light emitted 
by the laser, $a$ and $b$ - size of the laser beam on the surface of the photodetector, $\Delta x$ and $\Delta y$ - the position changes of the laser beam.

One should be aware, that presented formulas describe an ideal case, while the nonlinear behavior of the photodiode should be taken into account, in particular, when the shift of the laser beam in respect to the central part of the photodiode is used to calculate the probe's displacement. The approach aiming at solving this limitation was reported by Xie et al. [35]. The nonlinear calibration of the optical setup was performed for normal and lateral forces detection, after the spring constant for both directions was determined. Once the original force/position-voltage responses for the normal and lateral force calibration were acquired, the force/position-voltage responses to voltage-angular sensitivity responses were transformed. As a next step, the nonlinear fit voltage-angular sensitivity responses were employed to obtain continuous functions of and then calculate the angular sensitivity. Finally, the angular deflection could be calculated, enabling applied normal and lateral forces calculation. The procedure allowed to reduce the sensitivity error of the setup from $200 \%$ to $3.6 \%$. Also Nieradka et al. showed the method allowing to minimize the impact of the nonlinear response of the split photodiode [36] by utilizing simple function correcting unwanted detection distortion:

$$
u=A \arctan \left(B x^{3}+C\right)-D
$$

where: $u$ is the detector's output voltage, $x$ is the laser spot position, and $A, B, C$, and $D$ are the model parameters. The determination of the model parameters was performed by moving the laser spot over the surface of the photodiode and acquiring its response. Once the model was established, it allowed to perform the forces measurement in static mode with wide range of the forces and high sensitivity.

Due to the interest increase with the multi-probe systems, the solution based on extended OBD was implemented based on a single laser diode and set of two photodetectors [37]. It allowed to detect simultaneously the deflection of two probes without unwanted crosstalk.

A number of various issues must be considered while OBD is utilized. The stability of the laser beam, including the noise, is the major component limiting the detection sensitivity. In order to improve the light reflectivity (the most popular wavelength in optical detection systems is $640 \mathrm{~nm}$ ), the probes are covered with reflecting films $(\mathrm{Al}, \mathrm{Au})$ in a thickness range of 20-60 nm. The drawback of such a films is the change of the probe's mechanical properties (which has to be considered in case of theoretical and experimental calibration methods) [38] and in addition may introduce unwanted bending force due to different thermal expansion factors. This impact was discussed by Sader at al. [39]. Gibson et al. claimed however, that the film thickness up to $100 \mathrm{~nm}$ doesn't change significantly the mechanical properties of the cantilever [40].

It should emphasized, that optical detection method may also be a source of the unwanted modulation of detected signal, while the force spectroscopy measurements are carried out, and the probe is away from the surface. It is caused by the interference phenomena of the beams reflected from the cantilever and the surface. There are however commercially available setups with the lasers emitting the IR radiation (1300 $\mathrm{nm}$ ) to reduce an impact of such an issues [41]. Additionally, the limitations of the force detection have to be taken into account, in particular when measurements of very small forces are planned. A number of noise sources must be considered in case of OBD: shot noise of the photodetector, resistance Johnson-Nyquist noise in the $\mathrm{I} / \mathrm{U}$ converter, electronic circuits noise, laser instability noise, thermal noise of the probe. Efficient optimization of the system allows to improve the performance of the setup. Rode et al. presented the results of the modification of Bruker MultiMode V with Nanoscope V controller [42]. Obtained reduction of the deflection sensor noise density was approx. 1 order of magnitude (from 100 $\mathrm{fm} / \sqrt{\mathrm{Hz}}$ to around $10 \mathrm{fm} / \sqrt{\mathrm{Hz}}$ ).

The major advantage of OBD setup, is the simplicity in terms of fabrication and adjustment as well as relatively low cost of the probes due to the simple construction. The other optical detection technique, providing quantitative information about the probe's deflection, is the interferometry [43]. By utilizing optical fiber- based setup, one can relatively easily determine the deflection of the probe, however for the $\operatorname{sub} \lambda / 2$ travel, where $\lambda$ is a wavelength of radiation used in the setup, one should adjust setup in order to use the linear area of the detection signal in order to obtain the best possible detection dynamics and avoid the signal misinterpretation. For the travel larger than $\lambda / 2$, a special solution allowing the interference fringes count must be used. Such a setup requires a stable single-mode laser. The direct electrical readout of the probe's bending is relatively rare solution due to the costs of the probes caused by the complex fabrication process. Such a probes contain piezoresitors enabling the Wheatstone's bridge based deflection measurement [44, 45]. A series of successful implementations of the 1-D or 2-D matrix multi-probe setups were presented, enabling increased scanning trough output of the AFM [46, 47]. Moreover, such a probes can work in dark environment, therefore the advanced investigations of the optical-electrical properties of the material can be investigated.

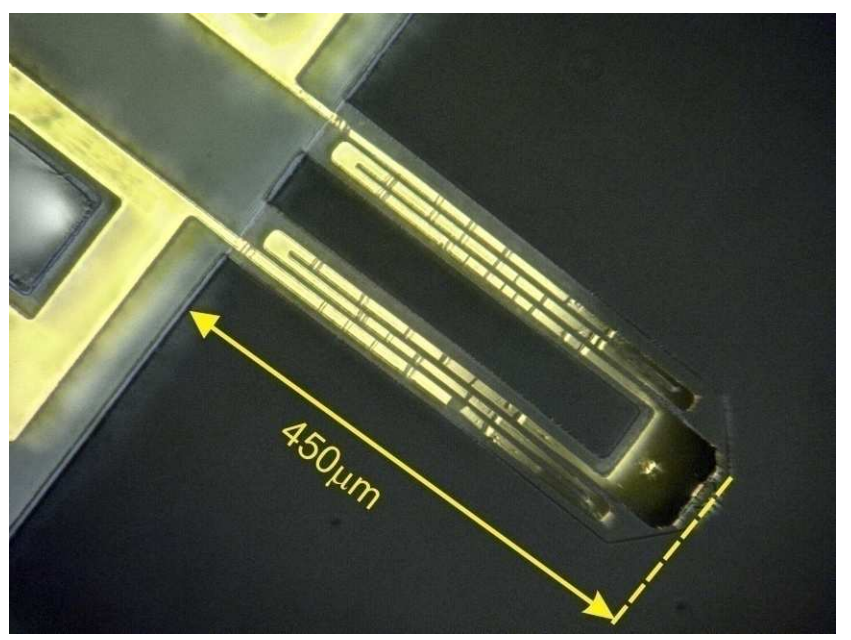

Fig. 5. The U-shaped polymer probe [48]. 
The implementation of the tensometer based detection was also implemented [48]. The changes of the resistance of chromium or gold deposited on the polymer microstructure of the probe. According to formula (7), the resistance changes are proportional to the elongation of the conducting paths:

$$
k=\frac{1}{\varepsilon} \frac{\Delta \rho}{\rho}+(1+2 v)
$$

where $\varepsilon=\Delta l / l, \quad l-$ length of the structure, $\rho-$ material's resistivity, $v$ - Poisson's constant. Two types: V-shaped and U-shaped probes (fig. 5) were developed and successfully tested.

Nanosensitive mechanical microprobes with CMOS transistors, inverters, inverter cascades and ring oscillators were presented by Łysko et al. [49]. As some of developed transistors were located in the area of the highest tension of the probes, the piezoresistive effect caused the changes of the resistance of the channels. Therefore the parameters of developed circuits were force-sensitive. High deflection sensitivity $(1.2-1.8 \mathrm{mV} / \mathrm{nm})$ and force sensitivity $(2.0-2.4$ $\mathrm{mV} / \mathrm{nN}$ ) was obtained. Cascade arrangement of the inverters bases on transistors, allowed to acquire the deflection sensitivity up to $40 \mathrm{mV} / \mathrm{nm}$. For ring oscillator probes, 5-8 $\mathrm{Hz} / \mathrm{nm}$ deflection sensitivity was determined. Such a solutions are compact and robust in terms of the application as the detectors for chemical or biological sensing.

One should also mention a unique solution of electromagnetically actuated probe with optical detection presented by Kopiec et al. [50]. The Halbach array providing approx. $330 \mathrm{mT}$ magnetic field allowed to cause the normal force acting on the probe, when the current was flowing through the conducting lines developed along the sides of the microstructure. This solution was used for detection of the SAM (Self Assembling Monolayers) presence on the surface of the probe. By the control of the current flowing through the electromagnetic actuating system, the deflection of the probe was maintained at that same position, while SAM was covering the surface of the probe. Therefore the driving current was proportional to the force caused by the SAM presence. The force acting on the probe for full coverage of its surface was $4 \pm 0.48 \mathrm{nN}$. The major advantage of this solution is, that the maximum sensitivity of the setup is provided at all times, as the laser beam is aligned at the center of the four section photodiode. Moreover, the thermal drift can be compensated using that same control fashion.

Also other techniques were demonstrated as efficient methods for the detection of the probe deflection, such a capacitance measurement [51] and tunneling current measurement [52]. However, due to a complicated handling, no practical utilization was recently reported.

\subsection{Positioning Systems, Data Acquisition and Processing}

In order to obtain the quantitative information about the tip-sample distance change, piezoactuators-based positioning systems with feedback are desired, as piezoactuators suffer a number of issues. A variety of displacement sensors is used: tension, capacitive, optical/intensity and optical/ interferometric [53-55]. One should be aware, that only the last one provide the distance readout directly. The other techniques require the calibration.

The final component of the setup is the signal $\mathrm{A} / \mathrm{D}, \mathrm{D} / \mathrm{A}$ conversion and processing unit. It includes hardware (DSP, FPGA or other microprocessor-based) solutions with advanced $\mathrm{A} / \mathrm{D}$ and $\mathrm{D} / \mathrm{A}$ converters $[56,57]$ and software [58-60]. The recent progress in terms of the development of electronics as well as programming enables simple, efficient and high-resolution data acquisition systems. One of the highest priorities taken into account during the system development is its stability and flexibility, as users demand reliable measurements as well as possible upgrading without physical modifications of system's construction. High quality $\mathrm{A} / \mathrm{D}, \mathrm{D} / \mathrm{A}$ converters provide the noise reduction, higher resolution and measurement performance. Also advanced laser control features may increase the stability of the data acquisition procedure [61].

\subsection{Force Spectroscopy Technique and Related Methods}

As mentioned earlier, the force spectroscopy technique is so far the most popular and common method applicable for the determination of the mechanical properties of the surface or investigation of the behavior of nanometer-scale objects. As the tip-sample distance decreases, the attractive and repulsive forces are experienced by the probe. The approach is continued until certain force is measured or distance travelled. In terms of the protecting the tip and sample against damages, the force limit feature should be used. Once the curve is acquired (fig. 6), a set of information can be obtained regarding to the experiment and tested object: the stiffness (R1), snap-in force (F1), snap-off (adhesion) force (F3), peak force (F2), energy dissipation for the tip-sample separation (E2), energy dissipation for the surface deformation (E1), elasticity, unbinding force, unbinding length and so one. The obtained information variety is very valuable in terms of the analysis of complex phenomena at nanoscale.

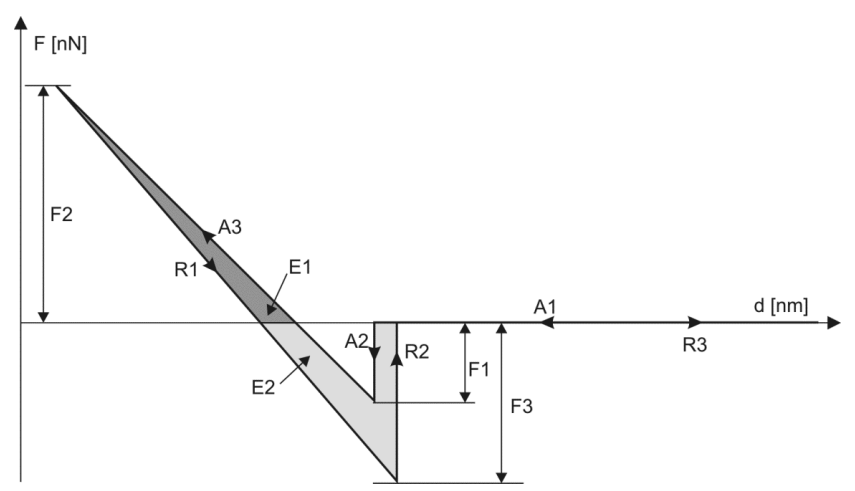

Fig. 6. The typical shape of the force spectroscopy curve.

It should be underlined, that single curve may be considered as very fragile in terms of random noises and unwanted events, therefore a series of few tens, hundreds or even thousands is typically acquired in order to provide the statistical outcome. It is however, still, the information concerning specific spot, 
which may be insufficient source of the information, as the spatial distribution of the properties and homogeneity must be often investigated. As the measurement is the time-consuming process, one has to consider carefully any speed increase in such kind of measurements, as some tip-sample interactions are speed-sensitive. This particular phenomena may be used experimentally in order to distinguish specific interaction mechanisms $[19,62]$.

In order to perform the mapping of the surface's mechanical properties, the Force Volume mode was developed [63, 64], enabling the acquisition of a series of the force spectroscopy curves at the nodes of grid covering previously scanned area. It was presented as efficient tool in investigation and was successfully utilized in investigations of biological or chemical samples. The major drawback of this approach is the imaging speed, as the time necessary to acquire the data with the resolution 512 x 512 would reach even dozens of hours. Therefore typically, this technique was used for imaging the mechanical properties with the resolution of $32 \times 32$ points. On the other hand, Jumping Mode Scanning Force Microscopy $[65,66]$ and Digital Pulse Force Mode [15, 67] were also proposed as techniques allowing to investigate the mechanical properties of the surface, however the mapping speed was still insufficient. To compensate the drawbacks mentioned before, another approach, described following section, was developed in order to provide high speed mapping of the mechanical properties of the sample.

Legleiter et al. presented Scanning Probe Acceleration Microscopy (SPAM) [68], where the oscillation signal of the probe working in semicontact was processed in order to extract the tip-sample interaction information, leading to determination the maximum interaction force, providing insight into the stiffness maps. However due to necessary processing time, the single scanning procedure could take up to several hours.

\subsection{Time-Resolved Tapping Mode Basing Techniques}

Unlike in force spectroscopy requiring specific approach/ retract ratio of the tip (typically in range of 10-1000 nm/s), which significantly limits the imaging trough output as well as abovementioned techniques, the time-resolved tapping mode approach bases on the high-ratio sampling of the tip-sample interaction. In this group of techniques the cantilever oscillates as in the intermittent contact regime, with the frequency in range from few $\mathrm{kHz}$ (Peak Force QNM mode from Bruker [69], QI from JPK [70] to tens of $\mathrm{kHz}$ (Harmonix [71, 72], NanoSwing [73, 74]). The force curves acquired with the speed of few thousands per second must be filtered, fitted, quantified and recorded by the data processing unit. Obtained information may contain: stiffness, Young modulus, adhesion force, energy dissipation for the tip-sample separation, energy dissipation for the surface deformation and peak force. Due the complexity and diversity of abovementioned methods, no detailed information about the acquisition and processing method is provided here. In general, various approaches were utilized: analysis of the torsional oscillations of the T-shaped probe (fig. 7), the longitudinal oscillation excitation at the frequency of few $\mathrm{kHz}$, significantly below the resonance frequency, or advanced phase shift and resonance quality processing. As obtained maps of specific properties are expected to contain quantitative data, both: the mechanical properties of the probe and tip-sample interaction model have to be determined. In general, due to the data acquisition and processing complexity, the calibration is performed using the reference samples provided with specific system.

It should be underlined, that T-shaped probe, used for time-resolved tapping mode, was also tested in terms of the lateral forces measurements. In order to obtain the quantitative data, Reitsma et al. presented the method of determination the torsional spring constant using designed support edge allowing to apply the torsional force at various distances from the symmetry axis of the probe [75].

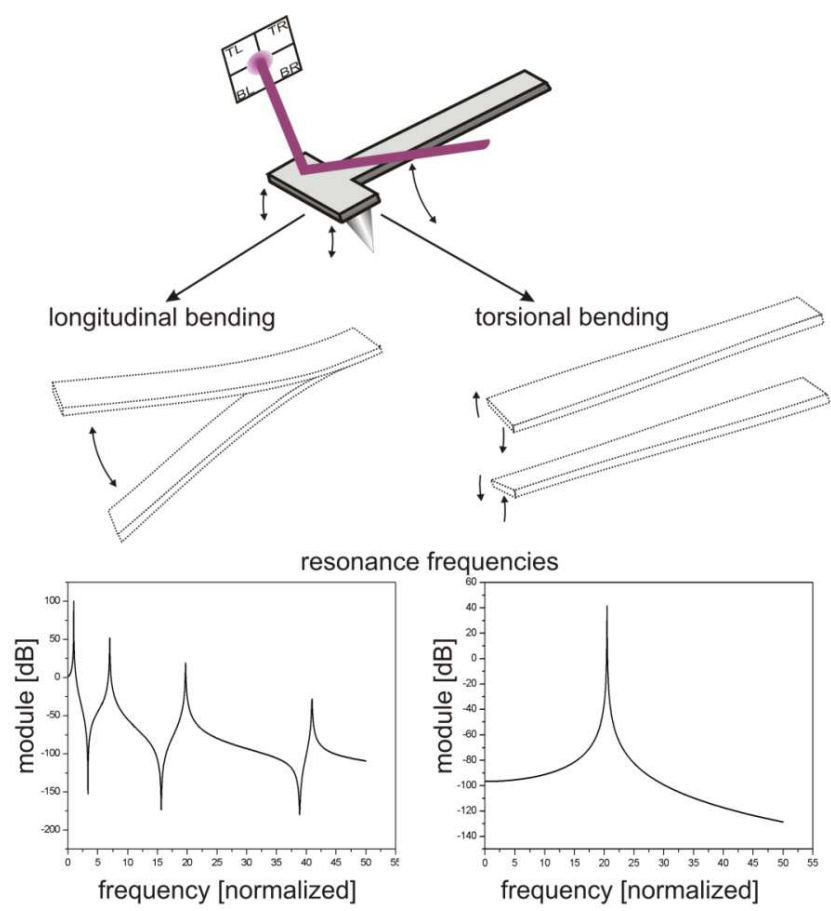

Fig. 7. The idea of utilization of torsional oscillations with T-shaped probe to detect wide bandwidth signals.

\subsection{Interaction Models}

The final step of the measurement process is the quantification of investigated parameters. In terms of determining of the mechanical properties of the surface, the force spectroscopy curve must be analyzed according to specific interaction model covering specific tip-sample interaction phenomena, present in certain case. There are five main models commonly used in the data analysis: Hertz, DMT, JKR, M-D and Bradley [76]. For the majority of the samples, DMT model is sufficient to determine for instance the Young modulus $[77,78]$. However, one should carefully consider the experiment conditions and the outcome, in particular: the elasticity and the adhesion as the calculation results are strongly correlated to used model [79-81]. 


\section{The Probe's Spring Constant Determination Methods}

Various approaches developed in order to determine the spring constant of the probes can be grouped in three main categories proposed by Clifford et al.: dimensional (theoretical), experimental static and experimental dynamic [82], Matei et al. used following classification: thermal, load and dimension [83]. Gibson et al. on the other hand used four categories [84]: dynamic, theoretical, static response and indirect methods.

A number of criterion has to be considered in terms of practical applications of the calibration method: accuracy, standard AFM setup compatibility, additional equipment demand (SEM, nanoindenter, micromanipulators, advanced software), procedure time consumption, user's expertise demand. Table 2 summarizes the key simplified factors of described below calibration solutions.

Table 2. The short summary of the spring constant calibration method.

\begin{tabular}{|c|c|c|c|}
\hline Method & Uncertainty & $\begin{array}{l}\text { Tip damage } \\
\text { risk }\end{array}$ & Complexity \\
\hline Theoretical model & $2-11 \%$ & None & Low \\
\hline Finite model analysis & $3-5 \%$ & None & High \\
\hline Thermal noise & $10 \%$ & None & Low \\
\hline Added mass - static & $15-25 \%$ & High & High \\
\hline Added mass - dynamic & $10 \%$ & High & High \\
\hline Sader method & $10-15 \%$ & Low & Low \\
\hline Vibrometry/ FEA & $2 \%$ & Low & High \\
\hline $\begin{array}{l}\text { Nanointentation } \\
\text { instrument }\end{array}$ & $5 \%$ & Medium & High \\
\hline Precision balance & $1 \%$ & High & High \\
\hline Cantilever on cantilever & $2-10 \%$ & High & High \\
\hline Calibration structures & $3 \%-10 \%$ & High & Low \\
\hline $\begin{array}{l}\text { Microforce sensing } \\
\text { micromanipulator }\end{array}$ & $3.2 \%$ & Medium & High \\
\hline Micropipette & $10 \%$ & High & High \\
\hline $\begin{array}{l}\text { Dynamic viscous } \\
\text { response }\end{array}$ & $4 \%$ & Medium & High \\
\hline Material reference & $50 \%$ & High & Medium \\
\hline Indirect method & $10 \%$ & None & Low \\
\hline
\end{tabular}

It should be emphasized, that the continues progress in development and application of scanning probe techniques allowed to achieve the level of maturity, which enabled international standards activity in order to provide specific conduct rules for the industry and science. The technical committee ISO/TC 201, sub-committee SC9, already provided standards concerning a basic issues of the scanning probe microscopy utilization such as: definition and calibration of the lateral resolution of a near-field optical microscope [85], measurement of the drift rate [86], calibration of measuring systems [87], and procedure for in situ characterization of AFM probe shank profile used for nanostructure measurement [88]. The issue of normal spring constant determination is recently under development and the document ISO/DIS 11775 [89].

\subsection{Theoretical Model}

In order to provide the easy and nondestructive method allowing to determine the spring constant basing on the specific parameters provided by the producer, one can calculate the spring constant basing on the physical dimension and the Young modulus. Several approximations of parallel beam approximation (PBA) were developed and tested [90-92]:

$$
\begin{aligned}
& k=\frac{E t^{3} w}{2 l^{3}} \cdot\left\{1+\frac{b^{2}}{4 L^{2}}\right\}^{-2} \\
& k=\frac{E t^{3} w}{2 l^{3}} \\
& k=\frac{E t^{3} w}{2 l^{3}} \cdot\left\{1+\frac{4 d^{3}}{b^{3}}\right\}^{-1}
\end{aligned}
$$

Sader et al. developed following formula for V-shaped probes $[39,92,93]$ :

$$
k=\frac{E t^{3} w}{2 l^{3}} \cdot \cos \theta\left\{1+\frac{4 w^{3}}{b^{3}}(3 \cos \theta-2)\right\}^{-1}
$$

while this formula basses on the assumption, that the force is applied to the end of the probe, simple equation allows to correct the outcome, taking into account the real point of the load (the tip location) [39, 92]:

$$
k_{c}=k(l /(l-\Delta l))^{3}
$$

where $E$ is the Young's modulus, $t$ is the thickness, $w$ is the width and $l$ is the length, $b$ is the total width of the base of the cantilever, $d$ is the width of single arm of the cantilever, is and $\Delta l$ is the distance between the tip and the end of the cantilever. The approximation error of presented models in formulas 8,9 , 10 and 11 is, respectively: $25 \%, 16 \%, 13 \%$ and $2 \%$ [92].

For rectangular probes formula 8 can be simplified to following form (Bernoulli beam equation):

$$
k=\frac{E t^{3} w}{4 l^{3}}
$$

Also in this case the correction formula (eq. 12) should be applied.

It should be underlined, that the main limitation of this method's accuracy is the Young's modulus determination precision. Also, the models assume shapes uniformity, as in case of the thickness, as critical parameter - it was shown that it may introduce a significant error [94, 95]. The model taking into account this particular issue was presented by Fretrup and Allen, where effective thickness $t_{\mathrm{es}}$ provides that same outcome that nonuniform probe where thickness profile is $h(x)$ [95]:

$$
\begin{gathered}
k=\frac{E t_{e s}^{3} w}{4 l^{3}} \\
t_{e s}=-\frac{1}{3}\left[\int_{0}^{1} \int_{0}^{\tilde{x}} \frac{(x-1)}{h(\tilde{x})^{3}} d \tilde{x} d x\right]^{-1}
\end{gathered}
$$

Additionally, the complexity of the probes (deposited layers) has an impact on the calculation result in terms of presence of additional mass [39, 40]. Moreover, the issue of stress introduced by multilayers can have an influence on calculation results [96]. 
The development of the theoretical models was presented by Neumeister and Ducker [97]. In mentioned study, the methods for calculation lateral/torsional, longitudinal and normal spring constant for $\mathrm{V}$-shaped probes were presented. The accuracy of this approach was approximately $11 \%$.

\subsection{Finite Elements Analysis (Finite Elements Method)}

The theoretical approach requires a specific knowledge of the probe's dimensions. In order to acquire such an information, the optical microscopy as well as the electron microscopy may be utilized. Such a choice is always the question of the compromise between the procedure's time consumption and accuracy of the measurement. Anyhow, the FEA method is considered as rather time consuming due to the calculation process. Additionally, any non-homogeneity of the shape of the cantilever or the material, leads to a significant error presence, which is difficult to fit, in particular, when various levels of the modeling complexity may be implemented. It should be underlined, that a number of approaches was developed, and different levels of accuracy were obtained. Clifford et al. [98] showed the modification of the solution proposed by Neumaister and Ducker [97] where match level of $1 \%$ was obtained. Also Chen et al. developed this particular approach [99]. They presented the impact of various factors on the calculation uncertainty such as: Poisson factor, the cantilever's thickness variation, mass of the tip, the tilt angle and presence of the non-axial mass causing torsional forces. Such an insight is useful in case of experimental techniques, as it allows to estimate the contribution of setup and conditions- related issues. The simulation outcome provides a knowledge allowing to develop the conduct of performing any experimental method in order to reduce possible calibration error.

Georgakaki et al. performed the calculation of the spring constant of the probes utilized for intermittent contact measurements [38]. Due to the fact, that typically such a cantilevers are relatively stiff (in range of 20-100 N/m), some of alternative techniques, such as thermal method are not suitable here. For modeling, various shapes of the cross section of the probe were implemented (rectangular, trapezoidal) and compared with the measurements performed using Sader method $[39,93]$. The modeling was carried out basing on the Cleveland's formula [100]. Approximately 7\% combined uncertainty was declared. Additionally, the impact of $\mathrm{Al}$ reflective coating in thickness range from 0 to $50 \mathrm{~nm}$ was taken into account. The calculations showed possible changes of the spring constant due to the metallic film presence up to $3.9 \%$.

Certain level of the model development and calculating essential phenomena, allows to use the FEM in practical applications in order to design the AFM probes according to specific applications. Gotszalk et al. presented [101] the development process of piezoresistive detection- based probes. During the modeling process the unique shape of the probe was optimized in terms of the specific application, and spring constant of the probe was determined. Also the location of the piezoresistors was optimized in terms of maximal sensitivity.
The Finite Elements Analysis was also performed by Loizeau et al., in order to predict the result of implementation additional V-grooves in the cantilevers, as the aim of the work was to increase the spring constant up to two orders of magnitude [102]. The essential factors development error was assumed to maintain below 5\% level.

\subsection{Thermal Noise Method}

Recently the thermal noise method is probably the most popular technique utilized during routine measurements. As no additional equipment is necessary and AFM control software often provides such functionality, the user can perform the operation without effort within just few minutes. The thermal noise method presented by Hutter et al. bases on the acquisition of the vibration of the cantilever caused by the thermal fluctuations. The Hamiltonian formula describing this phenomena is presented below [103]:

$$
H=\frac{p^{2}}{2 m}+\frac{m \omega_{0}^{2} q^{2}}{2}
$$

where $q$ is the displacement of the oscillator, $p$ is its momentum, $m$ is the oscillating mass and $\omega_{0}$ is the resonant angular frequency of the system. Furthermore, concerning equipartition theorem, the average value of each quadratic term in the Hamiltonian is given by $k_{B} T / 2$, where $T$ is the temperature and $k_{B}$ is the Boltzman's constant. Therefore:

$$
\left\langle\frac{1}{2} m \omega_{0}^{2} q^{2}\right\rangle=\frac{1}{2} k_{B} T
$$

Knowing that $\omega_{0}^{2}=k / m$, the spring constant can be determined from the mean-square displacement of the probe: $k=k_{B} T /\left\langle q^{2}\right\rangle$

At room temperature the probe of $0.05 \mathrm{~N} / \mathrm{m}$ will oscillate thermally with the amplitude of $0.3 \mathrm{~nm}$, which is relatively easy to detect if sufficient amount of data is acquired. However, due to presence of unwanted noise, the analysis of the signal in the frequency domain is necessary in order to provide efficient data filtering. By analyzing the power spectral density of acquired signal and approximation of obtained distribution with the Lorentzian curve, one can calculate the area of the power spectrum of the thermal noise, enabling the determination of the spring constant of tested probe:

$$
k=k_{B} T / P
$$

The main limitation of this method is the range of the spring constant, as for stiffer probes $(\mathrm{k}>10)$ it is difficult to detect the thermally induced oscillations. This limitation was noticed in other publications.

Butt and Jaschke verified the outcome of the thermal noise method, using various detection methods [104] such as optical interferometry, tunneling current they proposed the formula describing the thermal noise and the oscillation amplitude for rectangular cantilever:

$$
\sqrt{\left\langle q^{* 2}\right\rangle}=\sqrt{\frac{4 k_{B} T}{3 k}}=\sqrt{\frac{4}{3}\left\langle q^{2}\right\rangle}
$$


They also introduced the virtual deflection parameter $\left(q^{*}\right)$ as the result of the measurement using optical system, as they pointed out the drawbacks of this setup in terms of the measurement of real oscillation amplitude, such as detection of the angle of the end of the cantilever instead of the real vertical movement. The impact of this drawback increases for higher oscillation modes. Additionally, Butt and Jaschke provided the formula describing the virtual deflection for $i$-th vibrational modes [104]:

$$
\left\langle q_{i}^{* 2}\right\rangle=\frac{16 k_{B} T}{2 k \alpha_{i}^{2}}\left(\frac{\sin \alpha_{i} \sinh \alpha_{i}}{\sin \alpha_{i}+\sinh \alpha_{i}}\right)^{2}
$$

while the real oscillation amplitude is:

$$
\left\langle q_{i}^{2}\right\rangle=\frac{12 k_{B} T}{k \alpha_{i}^{4}}
$$

Levy and Maaloum proposed utilization of I and II oscillation modes in order to increase the accuracy of the method [105]. As the virtual height had to be taken into account, following formulas were used to determine the spring constant:

$$
\begin{aligned}
k & =0.82 \frac{k T}{\left\langle z_{1}^{* 2}\right\rangle} \\
k & =0.25 \frac{k T}{\left\langle z_{2}^{* 2}\right\rangle}
\end{aligned}
$$

for first and second oscillation mode respectively. Additionally, Levy and Maaloum compared the results obtained using the analysis of first and second oscillation mode as well, as the Sader's method. As six rectangular probes from the same wafer were tested, a significant variation of the spring constant could be also noticed. The outcome of the procedure varied in range: $16.8-22.9 ; 16.0-22.9 ; 18.0-22.7$ $\mathrm{N} / \mathrm{m}$ for first, second oscillation mode and Sader's method respectively. According to the manufacturer's documentation, $20 \mathrm{~N} / \mathrm{m}$ was the nominal value.

Allen et al. performed the experimental work focused at verification of the impact of the mass of the tip on the accuracy of the thermal noise method [106]. The extended models revealed a significant differences of the oscillation shapes of $1^{\text {st }}, 2^{\text {nd }}, 3^{\text {rd }}$ and $4^{\text {th }}$ oscillation modes for various probe/ tip mass ratios. Finally, the table of leading constant was provided, in order to use appropriate correction factor. In such a case, however, the knowledge concerning tip/ cantilever mass ratio is necessary.

Proksch et al. proposed further improvement of the method by taking into account the position of the laser spot along the cantilever [107]. They showed, that this factor may introduce the variation of the spring constant calculation up to $15 \%$ for short probes $(60 \mu \mathrm{m})$ and $50 \%$ for long probes $(225 \mu \mathrm{m})$. They also confronted the spring constant determination results with the method developed by Sader and Cleveland. The results systematically disagreed by $50 \%$ and $25 \%$ for short and long probes respectively. They proposed a new model taking into account the diameter and position of the laser spot, and as the result, the differences between used methods was reduced down to $10 \%$. In order to calculate the ODB setup sensitivity, one can use on-line calculator [108].

Heim et al. presented the utilization of the thermal noise method for determination of the spring constant of the colloidal probe cantilevers [109]. They used tipless bare probes as well as V-shaped cantilevers. Although the signal/noise ratio was lowered due to the added mass, no influence was found in terms of the accuracy. In the ideal cases, the spring constant agreed within $5 \%$ with the bare tipless cantilevers used for the preparation of the colloidal probes. However, the off-axis particles location could cause bare-colloidal probe difference increase up to $20 \%$ on average and up to $50 \%$ in worst cases, as the harmonic oscillator model becomes more complex in such a cases.

Boudaoud et al. verified the impact of the ground vibrations and acoustic noise on the thermal method, as it is very sensitive technique and presence of certain noise may be a source of significant fraction of error [110]. They showed, that at the temperatures $22^{\circ} \mathrm{C}$ and higher, the acoustic waves have more significant impact on the soft cantilevers $(\mathrm{k}<0.1 \mathrm{~N} / \mathrm{m})$, than the ground movement in particular, in noisy environment, where the acoustic noise exceeds $30 \mathrm{~dB}$ level at the $1 \mathrm{kHz}-20$ $\mathrm{kHz}$ bandwidth. Additionally, they presented the utilization of acoustic excitation of the cantilevers in terms of the spring constant determination. The error of the calculation increased from $2.42 \%(\mathrm{k}=0,0087 \mathrm{~N} / \mathrm{m})$, to $7.32 \%(0.03 \mathrm{~N} / \mathrm{m})$, therefore one can conclude, that acoustic excitation can be used as supporting one to the thermal method for soft probes.

Practical utilization of thermal method was presented by Nørrelykke and Flyvbjerg [111]. In this work, the AFM probes and optical tweezers (OTs) were analyzed in terms of recognition of practical similarities and differences, as the approach developed for the cantilevers could not be adapted straightforward to the OTs.

\subsection{Static Displacement with Added Mass}

Adding a mass to the end of the cantilever causes its deflection due to the gravity forces. It requires then very small objects, allowing to be placed easily on the top of the probe with high precision. Used object should be very regular in shape in order to calculate its volume, and made of the material (optimally single element) chemically stable, as its mass must be calculated with high degree of confidence. Senden and Ducker presented this method [97] and tested using 10-50 $\mu \mathrm{m}$ tungsten spheres. The formula allowing to calculate the spring constant was as follows:

$$
k=\frac{8 \pi R^{3} \rho g}{3 \Omega X}
$$

where: $\Omega$ is the deflection calibration, $g$ is the acceleration due to gravity, $\rho$ and $R$ are the density and the radius of the microsphere. $X$ is the distance of the probe's deflection, which in this case was doubled for the gravity, as the scanning heat was rotated $180 \mathrm{deg}$. This component of the equation introduces the largest uncertainty contribution: approx. 10\%.

Bonaccurso and Butt presented the solution, where the water drop on the surface of the probe is used in order to cause a set of various forces [112]. It allowed to observe few kinds of 
interactions: the weight of the drop placed at the free end of the probe provides its static bending proportional to the gravitational force. The kinetic energy of falling water drop provided the oscillation excitation, therefore the resonance frequency of such a setup could be determined. Moreover, the water drop placed next to fixed end, caused the probe's bending due to surface energy. The vaporizing of the water enables changed its mass and forces acting on the cantilever, therefore if the size/ volume of the drop was monitored with sufficient accuracy, the determination of the spring constant is possible. Basing on the formula presented by Cleveland at al. [113], the mechanical parameters of the probes could be determined.

$$
\begin{aligned}
f(t) & =\frac{1}{2 \pi} \sqrt{\frac{k}{m_{i}+0.243 M-\alpha t}} \\
f & =f_{i}+\frac{\alpha f_{i}^{3}}{2 k} t
\end{aligned}
$$

where $\alpha$ is the initial evaporation rate in $\mathrm{kg} / \mathrm{s}$. As the evaporation continues, the resonance frequency increases. By monitoring this process, one can extract the linear slope in order to calculate $\frac{\alpha f_{i}^{3}}{2 k}$ component. The water drop size was controlled with optical microscope, however precise calculation of its shape and generated force, requires consideration of few wetting phenomena.

\subsection{Added Mass Method / Dynamic}

Cleveland et al. presented approach basing on the analysis of the resonance frequency of the cantilever due to additional mass attachment to its end [113]. The formula describing the spring constant of the cantilever with rectangular cross is presented below:

$$
k=\frac{E t^{2} w}{4 l^{3}}
$$

where $E$ is the elastic modulus, $t$ is the thickness, $w$ is the width and $l$ is the length. For a uniform cantilever of a rectangular cross the effective mass is $m^{*}=0.24 m_{b}$ where $m_{b}$ is the mass of the beam. When an end mass $M$ is added, the resonant frequency is given by:

$$
\vartheta=\frac{\omega}{2 \pi}=\frac{1}{2 \pi} \sqrt{\frac{k}{M+m^{*}}}
$$

Therefore the spring constant may be determined using following equation:

$$
k=(2 \pi)^{2} \frac{M_{1}}{\left(1 / v_{1}^{2}\right)-\left(1 / v_{0}^{2}\right)}
$$

and effective mass is described with following equation:

$$
m^{*}=M_{1} \frac{v_{1}^{2}}{v_{0}^{2}-v_{1}^{2}}
$$

where $v_{0}$ and $v_{1}$ are resonant frequencies without additional load and with added mass $M_{1}$ respectively. In order to determine the resonance frequency no external excitation was used, as thermal noise provided large enough oscillations of the probe (approximately $1 \mathrm{~nm}$ of amplitude).

When a series of the measurements is performed for various added masses, the plot $k$ vs $\frac{1}{(2 \pi v)^{2}}$ is linear. This model was also used for V-shape probes, as in such a case it can be considered as the set of two parallel cantilevers.

The technique suffers however a major drawback: it requires the micrometer-size particles and micromanipulator with stereoscope allowing to place precisely the mass on the cantilever. The issue of attaching the mass the probe was solved by the natural adhesion (capillary forces) if the setup meets certain conditions (temperature, humidity and materials). On the other hand, Sader et al. could not relay on the capillary forces, and in order to stabilize the additional mass, glued it to the probe with the wax [39].

The main sources of the uncertainty are: the mass of the particles determination when both: weight or size estimation is used and the particle positioning precision. In total the accuracy of the method was estimated to $10 \%$.

Another solution was described by Gibson et al. [40]. By adding the thin gold film on the probe, the resonance frequency change was observed. The formula used in order to calculate the spring constant was as follows:

$$
k_{N}=(2 \pi)^{2} \frac{0.24 \cdot l \cdot w \cdot t_{A u} \cdot \rho_{A u}}{\left[1.02 v_{1}\right]^{-2}-\left[1.02 v_{2}\right]^{-2}}
$$

where 0.24 is the a correction factor required since the gold at the base of the cantilever contributes less to the effective mass than the gold at the end of the probe, $t_{A u}$ is the thickness and $A u$ is the density of the gold film and 1.02 is the correction factor taking into account the air damping in case of V-shaped probes.

In terms of the area of the additional mass location, Tseytlin developed kineostatic model of spring constant ratios with end extended mass [114]. Proposed approach allowed to perform the calculations for V-shaped probes at lower complexity level than FEA methods. It can be used in particular in case of the analysis of the higher modes of oscillations.

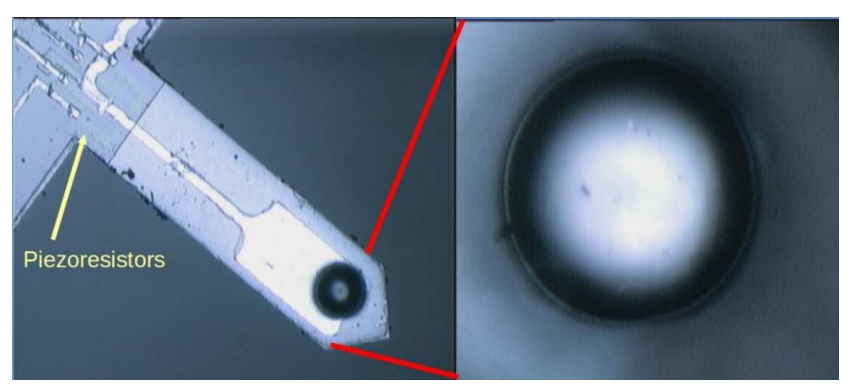

Fig. 8. The optical microscope view of AFM probe with piezoresistive bending detection and gold microsphere placed as added mass for spring constant determination [115].

Added mass method was used by Jóźwiak et al. for determination of the spring constant of the square- shape cantilever with piezoresistive bending detection system [115]. Despite complex construction and used materials diversity, the uniform mechanical properties of the microlever were assumed (silicon: $\rho=2.329 \mathrm{~g} / \mathrm{cm}^{-3}, \mathrm{E}=185 \mathrm{GPa}$ ). As an 
added mass object, the gold microsphere was used (fig 8). The mass of the microsphere was determined basing on the diameter measured by means of optical microscopy.

In general approach, added mass is removed after the spring constant calibration procedure is finished. Kopiec on the other hand presented the solution, where the resonance curve was measured before and after the golden microsphere was permanently attached as spherical probe [116]. Its diameter was measured using scanning electron microscopy. Obtained $37.46 \mu \mathrm{m}$ in diameter allowed to calculate mass $531.2 \mathrm{ng}$, and determine the spring constant of the probe to be $22.43 \mathrm{~N} / \mathrm{m}$. Additionally, EDS technique was used to verify the chemical purity of the microspheres, as the rapid melting of golden wire was used in the fabrication process. Due to the fact, that the microsphere was permanently attached to the probe in order to provide electrical conductivity, its position was stable and durable (fig. 9). As the result, the quantitative response of the electrostatic forces changes were observed, as various vapor phases such as: acetone or toluene were introduced to the spherical probe- surface setup.

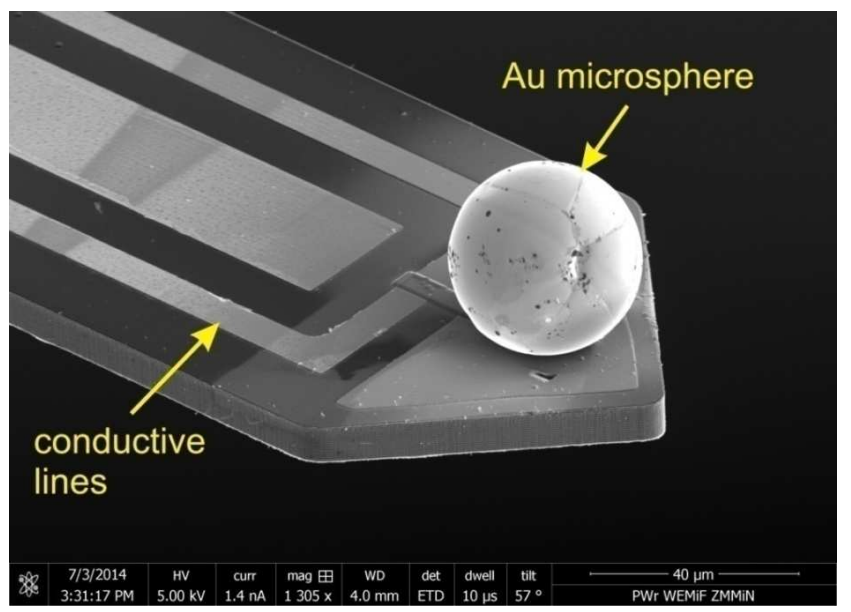

Fig. 9. The SEM view AFM probe with gold microsphere placed its surface as the probing feature as well as added mass for spring constant determination [116].

Bowen et al. presented the study, where the spring constant determination of various commercially available cantilevers, including $\mathrm{SiO}_{2}$ colloid probes, was performed by means of reverence cantilever and Sader method. In order to provide additional load, the probes were laminated with 3 and $8 \mathrm{~nm}$ thick Titanium and Chromium films by electron beam evaporation technique [96].

On the other hand, Woszczyna et al. performed the calibration of complex structures of the cantilever with integrated actuator and deflection detector [117] by removing some part of the probe by means of FIB (focused ion beam). The mass of removed tip was estimated to $1.31 \mathrm{ng}$. Such an approach may provide more accurate results, as the added mass position and mass-probe contact quality, as well as the added mass estimation (basing on the dimensions) can be determined with lower precision. Figure 10 shows the probe and its corners after the material was removed.

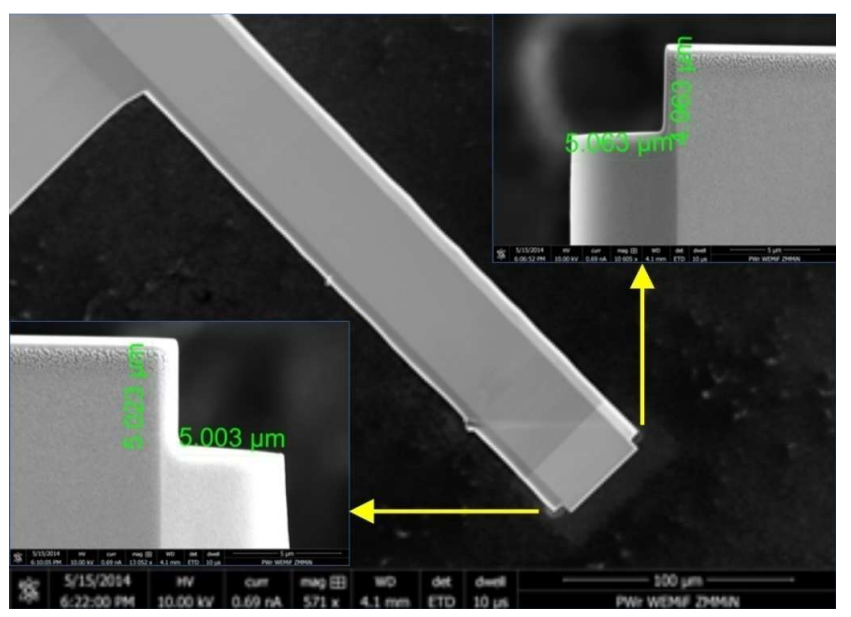

Fig. 10. The SEM view of the probe with removed corners by means of FIB [117].

\subsection{Sader Method}

The method basing on the acquisition of the resonance curve was proposed by Sader et al. [39, 93]. This approach allowed to reduce two major sources of uncertainty: the Young modulus and thickness of the cantilever. In order to measure mechanical response of the probe, the parameters of the environment, determining the behavior of the cantilever, should be known. Therefore, the measurements should be performed in the fluid, where complex hydrodynamic function $\Gamma(\omega)$ is determined.

$$
k=0.1906 \rho_{f} b^{2} L Q_{f} \Gamma_{\mathrm{i}}\left(\omega_{f}\right) \omega_{f}^{2}
$$

where $\rho_{f}$ is the density of the fluid, $Q_{f}$ is the quality factor of the fundamental oscillation mode in fluid, $\Gamma_{\mathrm{i}}\left(\omega_{f}\right)$ is the imaginary part of the complex hydrodynamic function $\Gamma(\omega)$ in fluid, $L$ and $b$ are the length and width of the cantilever and $\omega_{f}$ is the resonance frequency in fluid. $\Gamma$ depends on the Reynolds number

$$
R e=\rho_{f} \omega b^{2} / 4 \eta
$$

where $\eta$ is the viscosity of the surrounding fluid. This approach should provide the accuracy of the order of $10-15 \%$. Additionally, Sader analyzed significance of the presence and thickness (up to $70 \mathrm{~nm}$ ) of the reflective layer.

It should be noted, that due to simplicity of this method, relatively high accuracy and low tip damage risk, a number of works aimed at both: spring constant methodology determination development and measurement of mechanical properties of various samples were published [38, 84, 118-121]. Additionally, this approach was successfully utilized in air.

\subsection{Combined Method: Scanning Vibrometer and FEA}

A hybrid solution was presented by Mendels et al. basing on the analysis of the probes oscillations profiles, acquired with vibrometer at base and higher resonance frequencies in the vacuum [122]. Obtained data was combined with finite elements modeling, in order to resolve the physical properties 
of the probe. The profiles of the oscillation amplitude, acquired along the main symmetry axis of the probes as $3 \mathrm{D}$ maps. This approach allowed to obtain not only information about the longitudinal oscillations, but also about the torsional bending. Filtered data was correlated with FEA results. The tabulated properties of the silicon were adjusted in order to obtain certain level of correlation between the calculation and the measurement. The correction of the mechanical properties of the material in range of $0.5-0.01 \%$ increased the quality of simulation results. Finally, obtained agreement between all resonant frequencies was better than $1 \%$ with the FEA model and $2 \%$ with Timoshenko equation. Despite the fact that the effectiveness of the method was demonstrated in the vacuum, it may be however also applied in both: liquid and air.

\subsection{Nanoindentation Instrument}

Utilization of the well developed and easy to calibrate devices to determine the spring constant of AFM probes has a major advantage in terms of the traceability providing. Holbery and Eden presented the application of nanoindentation machine allowing to perform relatively easily direct unit transfer to the cantilever, as nanoindenters are equipped with precise force and displacement sensors [123, 124]. It should be underlined, that the calibration range is limited by the smallest measured forces of used equipment. Holbery and Eden obtained the accuracy better than $10 \%$ for the spring constant greater than $1 \mathrm{~N} / \mathrm{m}$. As typically utilized tips in nanondetation machines are too large to precisely apply the force to the cantilever, a etched tungsten wires can be used instead. It has to be underlined, that the precision location of applied force is an issue and it may introduce a significant error component.

Cliford and Seah demonstrated the uncertainty reduction while the nanoindentation device is utilized for spring constant determination, by performing the calibration procedure at few spots of the cantilever in order to be able to acquire the probe's response for various load forces [82]. They demonstrated that the total uncertainty may be reduced down to $5 \%$ if the force calibration of the nanoindenter is better than $1 \%$, and the calibration is performed at two spots on the probe at a distance 50 and $120 \mu \mathrm{m}$, and at each point 20 force-distance curves should be measured.

\subsection{Precision Balance}

Development of electrostatic balance, as the tool for calibration of the AFM probes (nano force calibrator - NFC) was presented by Kim et al. [125, 126]. As electromagnetic force compensation bases on basic physical phenomena, one can relatively easily provide the SI traceability of developed system. Direct force and distance control allows to perform direct unit transfer. The calibration setup allows to perform the procedure in wide range of the spring constant: lower than 0.1 $\mathrm{N} / \mathrm{m}$ and higher than $10 \mathrm{~N} / \mathrm{m}$. Another advantage of presented solution is the uncertainty, which was declared to be smaller than $1 \%$. In order to confirm unique metrological properties, long term measurements were performed revealing its high stability and repeatability. The setup was tested with various probes: piezoresistive cantilever as well as standard probes for contact and semicontact techniques.

\subsection{Calibration Cantilever (COC-Cantilever on Cantilever)}

The cantilever, once its mechanical properties are well defined, can be used as the reference object to calibrate AFM probes. Therefore, by using complex and sophisticated method, a cantilever can be calibrated with high accuracy to transfer the unit. Gibson et al. [127] proposed utilization of the cantilever of known spring constant to determine the parameters of unknown probe. As static load of one probe against the other was applied, their bending due to the acting forces allowed to transfer the mechanical properties to the unknown cantilever. The spring constant of the reference probe (V-shaped lever) was determined using Butt equation. The absolute accuracy was in range $\pm 20-30 \%$.

Gates and Pratt presented extremely uniform cantilevers providing the reference allowing to calibrate the AFM probes or other micromechanical devices $[128,129]$. The variety of the spring constant in range from 0.02 to $0.2 \mathrm{~N} / \mathrm{m}$ was obtained by fabrication of set of the cantilevers of different length from 300 to $600 \mu \mathrm{m}$. The structures were developed using SOI (silicon on insulator). In order to calibrate fabricated features, the electrostatic force balance (EFB) was used. It allowed to obtain the uncertainty better than $2 \%$ for the spring constant $0.02 \mathrm{~N} / \mathrm{m}$. The calibration outcome obtained during the procedure was compared with the calculations made using Euler Bernoulli equation. The agreement was within $3 \%$.

Slattery et al developed the cantilevers allowing to protect the tips of calibrated cantilevers against the risk of wear during the calibration procedure [130]. As the reference cantilever was equipped with the tip and probe being calibrated contained the nanomarkers fabricated using FIB (focused ion beam), it was possible to perform the surface imaging of the calibrated probe with the reference cantilever in order to locate the nanomarker and perform the procedure at this specific location. Although, in case of $\mathrm{V}$-shaped probes, one can set the load point at the arms intersection, therefore no additional topographical features are necessary. As the result, the major uncertainty source related to the load positioning issue could be minimized. The formula allowing to calculate the spring constant of the calibrated probe can be expressed as follows:

$$
k_{\text {test }}\left(L(L-\Delta L)^{-1}\right)^{3}=k_{\text {rigid }}\left(S_{\Delta L} S_{H}^{-1}-1\right) \cos ^{2} \alpha
$$

where $L$ is the length of the cantilever from the base to the tip, $\Delta L$ is the distance from the end of the calibrated probe to the point of the load applying, $S_{\Delta L}$ is the sensitivity of the cantilever at the position $\Delta L, S_{H}$ is the deflection detection sensitivity and $\alpha$ is the angle between the planes of reference and calibrated cantilevers.

Another approach was presented by Shaw et al. [131]. By using advanced methods such as indenter machine and 
electrostatic force balance (EFB), the spring constant of the reference probe was determined. This approach enables traceability of the AFM system, as the unit can be transferred from the devices present in the SI unit system metrological chain. The accuracy may be as good as $2 \%$, and it is mostly limited by the positioning precision of the reference and calibrated cantilever against each other, as the load point has major impact on the outcome. Additionally, the tip of calibrated probe may wear during the procedure. Moreover, the reference cantilever should have similar spring constant as the calibrated one, unless the design of the reference probe allows to perform the procedure at different its lengths.

One of the methods in terms of the transferring the force standards using static method for the spring constant determination was presented by Langlois et al [132]. The COC technique was extended, as the reference cantilever was equipped with piezoresistive force detection system. Therefore, once it was calibrated using EFB device, it was possible to correlate certain load force with the electrical response of the detection system connected to the probe. In order to confirm the performance of used method, seven cantilevers were calibrated using developed feature and compared to the results of the calibration performed using Sader method, thermal noise technique, nanoindetation and EFB. The range of nominal values of spring constant was from 0.2 to $40 \mathrm{~N} / \mathrm{m}$. The accuracy of presented method was estimated to be in range $\pm 5 \%$ to $\pm 10 \%$. It could be noted, that for soft probes the obtained values were in good agreement, but for stiff probes $(40 \mathrm{~N} / \mathrm{m})$ the outcome obtained with presented technique as well as thermal method deviated significantly from other results, proving limited range of application.

Cumpson et al. developed the $1.6 \mathrm{~mm}$ long and $150 \mu \mathrm{m}$ wide cantilever designed specifically for the standard transfer [133]. The specific features of C-MARS (cantilever microfabricated array of reference springs) allowed to place calibrated probe at specific positions due to the presence of binary markers along the C-MARS. Therefore one of the major uncertainty sources have been minimized. The access to 26 points along the feature, enabled the possibility of utilization reference spring constant in range from 0.0324 to $26.9 \mathrm{~N} / \mathrm{m}$. Additionally, the piezoresitors structure have been developed near to the base of the cantilever, which allows to monitor its bending or/and vibrations. Due to specific design, a limited range of calibration methods were available in order to calibrate C-MARS. Authors determined its spring constant using Euler-Bernoulli equation, finite element analysis and performed the calibration with Sader method in vacuum. Both theoretical methods delivered similar outcome $(0.041$ and $0.042 \mathrm{~N} / \mathrm{m}$ ), however experimental approach gave significantly smaller value $0.032 \mathrm{~N} / \mathrm{m}$. As the thickness of the cantilever is essential in terms of accuracy of theoretical calculations, it is likely that real value was $8 \%$ smaller than assumed $3 \mu \mathrm{m}$, therefore experimentally obtained value differed so much. The equation allowing to calculate the spring constant of tested probe $\mathrm{k}_{\mathrm{c}}$ using C-MARS, is as follows:

$$
\frac{k_{c}}{k_{r e f}}=\left[\frac{\left(\frac{\Delta V_{\text {stiff }}}{\Delta Z_{\text {stiff }}}\right)}{\left(\frac{\Delta V_{C-M A R S}}{\Delta Z_{C-M A R S}}\right)}\right] \sec \theta
$$

where $\frac{\Delta V_{\text {stiff }}}{\Delta Z_{\text {stiff }}}$ is the slope of the force spectroscopy curve acquired on the rigid surface, $\frac{\Delta V_{C-M A R S}}{\Delta Z_{C-M A R S}}$ is the slope of the force spectroscopy curve acquired on the C-MARS structure, $k_{r e f}$ is the spring constant of the C-MARS structure at the location the calibration is performed and $\theta$ is the angle of the calibrated cantilever in respect to the surface.

Despite the fact, that one can avoid the risk of the tip wear during calibration procedure, the sensitivity of detection system has to be determine in the first place. The procedure bases on pressing the tip against rigid surface, while the response of the detector is acquired. Tourek et al presented the solution, basing on the sharpened tungsten wire, allowing to avoid the contact of the tip with the surface [134]. One has to be aware, that the changes of the support point of the cantilever has an impact on its bending, therefore the sensitivity at the location of the tip $\mathrm{S}_{\mathrm{a}}$ can be calculated from following formula:

$$
S_{a}=\left[\frac{a_{f}^{3}}{w_{f}\left(2 a_{f} l_{f}-l_{f}^{2}\right)}\right] S_{w}
$$

where $S_{w}$ is the sensitivity measured at the contact point of the tungsten tip and cantilever, $a_{f}$ is the position of the tip, $w_{f}$ is the tungsten wire contact point and $l_{f}$ is the laser beam position. It was shown, that presented approach may introduce the sensitivity determination error up to $12 \%$.

\subsection{Calibration Structure}

A similar approach to the COC, is the method basing on utilization of specifically designed calibration structures allowing to determine the spring constant of the AFM probe with high accuracy, but easier than in case of the reference cantilevers.

Cumpson et al. developed compact feature providing the traceable unit transfer $[135,136]$. The device called MARS (microfabricated array of reference springs) is a complex, spring-like, planar feature made using micromachining processes, including chemical vapour deposition (CVD), wet etching, deep reactive ion etching. The set of the structures provided the spring constant references in range from 0.16 to $11.10 \mathrm{~N} / \mathrm{m}$. The accuracy of the spring constant determination provided with this approach is better than $5 \%$. However, due to small diameter $(50 \mu \mathrm{m})$ of the tip-feature contact area, practical utilization of presented solution requires a certain experience.

The micromechanical device, called T-MARS (torsional MARS) providing the reference spring constant in range from 0.01 to $4 \mathrm{~N} / \mathrm{m}$ was presented by Cumpson et. al [137]. The design of the feature bases on the suspended torsional spring structures. The advantages of T-MARS is relatively large surface of AFM tip landing, low resonance frequency and approximately $10 \%$ precision of the spring constant 
determination. Therefore the drawbacks of MARS were eliminated. The formula allowing to calculate the spring constant of calibrated probe is shown below:

$$
k_{c}=\frac{k_{\theta} \sec \theta}{d^{2}}\left[\frac{\left(\frac{\Delta V_{\text {stiff }}}{\Delta Z_{\text {stiff }}}\right)}{\left(\frac{\Delta V_{T-M A R S}}{\Delta Z_{T-M A R S}}\right)}-1\right]
$$

where $\frac{\Delta V_{\text {stiff }}}{\Delta Z_{\text {stiff }}}$ is the slope of the force spectroscopy curve acquired on the rigid surface, $\frac{\Delta V_{T-M A R S}}{\Delta Z_{T-M A R S}}$ is the slope of the force spectroscopy curve acquired on the T-MARS structure, $k_{\theta}$ is the local spring constant of the T-MARS structure at the location the calibration is performed, $d$ is the distance of the tip-structure contact point from the rotation axis of the structure and $\theta$ is the angle of the calibrated cantilever in respect to the surface.

Wagner et al. presented the method of determination of the effective spring constant using suspended silicon microbridge feature, in terms of utilized the probes in contact resonance AFM (CR-AFM) measurements [138]. In this technique the tip oscillates in this eigenmode while it maintains in the contact with the sample. Therefore the effective spring constant of the bridge is the essential parameter to determine. The data was obtained using two techniques: static force-distance curves acquisition and CR-AFM. The stiffness of the microbridge was calculated using Euler-Bernoulli model:

$$
k_{b}\left(x_{b}\right)=\frac{3 E_{b} I_{b} L_{b}^{3}}{\left(x_{b}\right)^{3}\left(L_{b}-x_{b}\right)^{3}}
$$

where $L_{b}, I_{b}$ and $L_{b}$ are Young modulus, bending moment of inertia and length of the bridge. Additionally, the optical detection performance of the probe's oscillation was verified in terms of the laser beam positioning on the probe. Moreover, performed experiment provided a practical guidance in terms of the resonance frequency adjustment.

Ekwińska et al. presented calibration features developed at millimeter-scale, providing the resistance to the unprofessional handling [139] (fig. 11). The utilization of the calibration features is relatively easy and does not require any complex procedure or additional equipment.

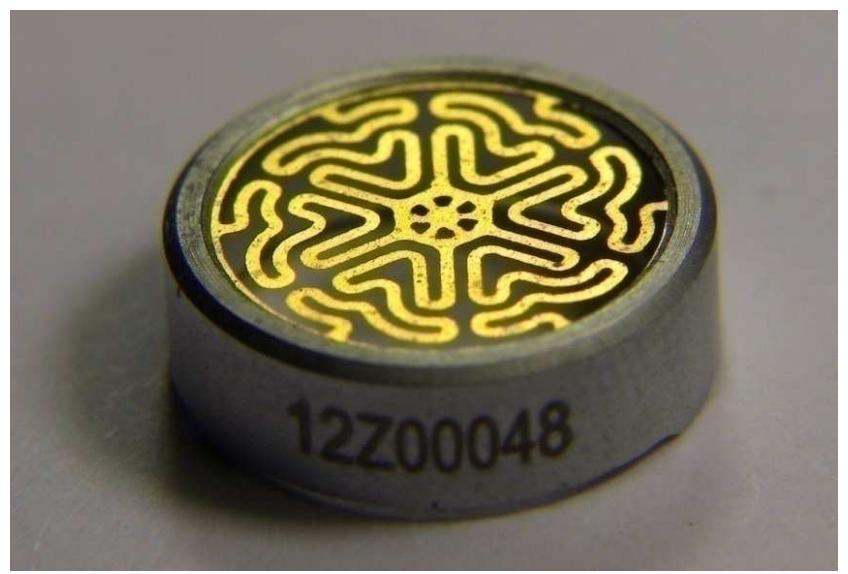

Fig. 11. The view of the calibration feature [139].
The operator has to acquire the force spectroscopy curves on the stiff surface (for instance the silicon) and on the calibration structure. As the mechanical properties of the structure are known (metrological traceable), the force constant can be calculated using the software provided by the producer [140-142]. It should be emphasized, that in order to obtain the highest possible accuracy, one should use the calibration feature with appropriate spring constant. In general, following rule should be fulfilled: $0.3 \mathrm{k}_{\mathrm{s}}<\mathrm{k}_{\mathrm{p}}<3 \mathrm{k}_{\mathrm{s}}$ where $\mathrm{k}_{\mathrm{s}}$ is the spring constant of the calibration feature, and the $\mathrm{k}_{\mathrm{p}}$, is the spring constant of the probe. Complete procedure takes typically less than 10 minutes. The uncertainty of the spring constant determination using those features can be lower than $\pm 3 \%$.

\subsection{Microforce Sensing Micromanipulator}

Unique approach of utilization of micromanipulator as the load for calibration AFM probes was described by $\mathrm{Li}$ et al. [143]. Presented micromanipulator was rectangular cantilever beam made of monocrystalline silicon and a tungsten cylindrical bar. In the silicon cantilever the piezoresistors were implemented providing the force sensing. Due to the size of the silicon bar: $0.95 \mathrm{~mm}, 0.15 \mathrm{~mm}$ and $5.0 \mathrm{~mm}$ in width, thickness and length respectively, the sharpened tungsten wire allowsed to operate with such small objects as AFM probes. In order to obtain quantitative force-electrical response correlation, the interferometric techniques have been utilized, as the displacement sensor, while the load was applied to the end of the tungsten wire. Once the microsensing manipulator was calibrated, it could be used to characterize the AFM probes.

Two microforce sensors have been developed and tested: $37.3 \mathrm{nN} / \mathrm{mV}$ and $705.4 \mathrm{nN} / \mathrm{mV}$. The tests allowing to perform the spring constant calibration of AFM have been carried out for three various cantilevers in range from $0.46 \mathrm{~N} / \mathrm{m}$ to 23.3 $\mathrm{N} / \mathrm{m}$. Covering such wide range of spring constant reveals large potential of demonstrated micromanipulators. The uncertainty of described method is $3.2 \%$.

\subsection{Micropipette}

Utilization of hydrodynamic pressure as the controlled force source was presented by Liu et al. [144]. The method called micropipette aspiration technique (MAT) bases on the utilization of the setup containing the micropipette with fitted closely spherical object such as polystyrene bead or cylindrical cell and fluid filling the micropipette. According to the fluid mechanics principles, a certain pressure acting on the sphere corresponds to a free motion velocity, under which the force is equal to zero. It provides piconewton precision, therefore it is suitable for the AFM probes calibration. Once the tested cantilever is aligned in order to touch the microsphere with the tip, the force is controlled by the liquid speed, and the deflection of the probe is measured optically, with support of two-dimensional nanotracking algorithm. The spring constant can be calculated using following equation: 


$$
k=\frac{\pi \alpha D_{p}\left(4 D_{b}-D_{p}\right)}{12 \dot{L}}
$$

where: $\alpha$ is pressuring rate, $D_{p}$ and $D_{b}$ micropipette and transducer diameter respectively, $\dot{L}$ is the time derivative of $L$ and it represents the cantilever-tip velocity. For described approach, the uncertainty is $10 \%$. In the experiment, successful calibration of the set of 33 probes was performed. In order to verify its performance, it was compared to the results of thermal noise method outcome. For the spring constant in range from 0.01 to 0.5 (nominal), the agreement between MAT and TN was better than $36 \%$.

\subsection{Dynamic Viscous Response}

One of the unique solutions of the cantilever's spring constant determination is utilization of the hydrodynamic drag force [145]. In order to determine the mechanical properties of the probe, its response to the forces acting on attached colloidal particle was observed. The particle was exposed to a oscillatory motion of a flat plane in a fluid medium. Therefore one should be aware, that this approach is limited to the colloidal probes $5 \mu \mathrm{m}$ in diameter or greater. The experimental setup was arranged using commercial AFM, after performing a number of modifications. The experiment was carried out as follows. After the probe approached the surface, it was covered with thin film of liquid placed in piezotransducer. Once the sinusoidal signal was applied to the transducer (typically $2-10 \mathrm{~nm}, 1 \mathrm{kHz}$ ) the drag force caused by the hydrodynamic interaction could be detected with typical ODB system.

The spring constant was calculated using the equation:

$$
k=\frac{1}{D} 12 \pi^{2} R^{2} \eta v\left[\left(\frac{A_{0}}{A}\right)^{2}-1\right]^{-\frac{1}{2}}
$$

In order to determine $A$, following formula was used:

$$
A=\left|A_{0}-x_{0} e^{i \theta}\right|
$$

where: $\eta$ is solution viscosity, $D$ is the mean particle-surface separation, $R$ is the particle radius, $v$ is the frequency of oscillation, $A_{0}$ is the drive amplitude, $A$ is the amplitude of the gap separation, $x_{0}$ is the amplitude response of the probe and $\theta$ is the phase shift between the drive signal and the measured oscillatory response. The practical utilization of presented method in terms of the calibration of AFM probes was also demonstrated. The accuracy of this method was estimated to maintain in range of $4 \%$.

\subsection{Material Reference}

Utilization of the material samples for the calibration of the AFM instrument in terms of quantitative imaging of the mechanical properties of the sample is very useful in terms of the techniques, where the signal acquisition and processing complexity utilized in used mode is too advanced for average user. Therefore, once the sample is scanned and its parameter is provided, the software adjusts the calibration factors in order to enable appropriate imaging. At least two various materials are necessary in order to provide required calibration accuracy [146]. It should be underlined, that due to uncontrolled ageing process and material non-homogeneity, the accuracy of this method is very difficult to estimate. It is however possible, to perform the verification of the properties of the reference material, using typical force spectroscopy [142]. It should be underlined, that adhesion forces measured with time-resolved technique and force spectroscopy method can't be compared, as this phenomena is speed related and obtained results may naturally differ due to physical phenomena [19]. The accuracy of this approach is related to many factors, however it unlikely may reach level better than $50 \%$.

\subsection{Indirect Method}

Indirect method is, as a matter of fact, a combined approach of any experimental technique, basing on an assumption of high repeatability of the parameters of the cantilevers fabricated during single process (same wafer). Gibson et al. presented indirect method basing on the assumption, that in case of utilized multiprobe chip, the material properties and the thickness of the probes in that chip are identical [147]. Therefore the calibration of one cantilever would provide sufficient data to calculate the spring constant of the other cantilevers with accuracy of the order of $10 \%$ or better. However, the work presented by Frentrup and Allen showed that the thickness of the probe may vary along the probe, introducing a significant error while the determination bases on the assumption of perfect shapes of the probe [95]. Moreover, as presented by Webber et al. the probes fashioned from that same wafer may differ even by factor of two [148], as the $101 \mathrm{~V}$-shaped probes made of silicon nitride was measured using thermal spectrum method proposed by Hutter and Bechhoefer.

\section{Further Issues}

Practical utilization of a number calibration methods, requires maintaining specific regime, providing the measurement conditions suitable to the assumption and simplification of the model used for the specific formula development. When any deviation from the optimal conditions occurs, one has to take into account the appearance of the unknown error of the spring constant determination. One of the factors, that one can easily overlook or be unable to optimize, is the scanning tip-surface angle. An interesting analysis of the influence of the complexity of the forces acting on the tilted scanning tip, causing the torque, presented Scott et al. [149]. As the perfect alignment the axes of the tip and normal to the surface is very difficult, some unwanted component forces can appear. Therefore one has to aware their influence on the effective spring constant, which may be increased or decreased. This conclusion extended the previous study's outcome [150], where only the enhancement of the effective spring constant was considered. Scott et al. provided the set of correction formulas for both: sharp tip and sphere ended probes, in order to take into account the impact of the analyzed phenomena on the final outcome. Although in 
typical conditions the value of correction factor may be barely negligible, in case of 45 degrees skewed sphere probe, it may reach $20 \%$ of the final value.

Once the variety of various spring constant determination methods was developed, one is interested in the outcome, when certain group of probes is calibrated using several methods. One has to keep in mind, that it is essential to provide specific conditions, allowing easily compare the obtained values.

The non-uniformity of the cantilevers was analyzed by Frentrup and Allen [95]. As theoretical calibration approaches assume the certain level of the regularity of the shapes of the cantilever, it can lead to a significant spring constant estimation error. In particular, the thickness deviations along the main symmetry axis of the probe may be significant (even few hundreds of percents of nominal value), therefore the oscillation modes may have different shapes than in the theoretical considerations. As the consequence, the ratios variations $\omega_{2} / \omega_{1}, \omega_{3} / \omega_{1}$ and $\omega_{4} / \omega_{1}$ may reach $17 \%, 21 \%$ and $24 \%$ respectively. On the other hand, when the thickness profiles of the cantilever are known, it is possible to correct the models in order to perform the spring constant calculation with the accuracy of few percent. Authors showed, that for Sader method, the typical non-uniformity influence can be rather small when the first vibration mode is utilized, but correction approach is effective up to fourth mode. On the other hand, for thermal tune method the non-uniformity has a serious impact, therefore one should not rely on the higher modes, as the correction formula is ineffective. The obtained errors for various correction methods are presented in table 3.

Table 3. The results of the spring constant determination obtained by Frentrup and Allen [95].

\begin{tabular}{lllll}
\hline Sader's metod & & & & \\
\hline ks & Mode 1 & Mode 2 & Mode 3 & Mode 4 \\
Uniform No Tip - Measured & $-2.22 \%$ & $2.62 \%$ & $6.24 \%$ & $14.23 \%$ \\
Uniform + Tip - Measured & $-2.71 \%$ & $4.14 \%$ & $15.91 \%$ & $25.38 \%$ \\
Linear and Lumped - Measured & $-0.06 \%$ & $5.91 \%$ & $10.52 \%$ & $15.43 \%$ \\
Linear and Lumped \#2 - & $-0.22 \%$ & $6.00 \%$ & $9.66 \%$ & $14.09 \%$ \\
Measured & & & & \\
Thermal tune method & Mode 1 & Mode 2 & Mode 3 & Mode 4 \\
ks & $15.31 \%$ & $72.79 \%$ & $160.93 \%$ & $287.70 \%$ \\
Uniform No Tip - Measured & $22.53 \%$ & $71.88 \%$ & $134.93 \%$ & $214.03 \%$ \\
Uniform + Tip - Measured & $6.32 \%$ & $13.82 \%$ & $35.79 \%$ & $73.20 \%$ \\
Linear and Lumped - Measured & & & & \\
Linear and Lumped \#2 - & $4.64 \%$ & $8.87 \%$ & $28.21 \%$ & $63.07 \%$ \\
Measured & & & & \\
\hline
\end{tabular}

Ohler performed the tests in order to compare the outcome of various methods, using the laser Doppler vibrometry (LDV) in order to improve the detection of the oscillations of the probes [121]. Three cantilevers: $400 \mu \mathrm{m}, 200 \mu \mathrm{m}$ and $100 \mu \mathrm{m}$ of length were tested using thermal tune technique, frequency scaling and Sader method. Detection of the probe's oscillation was carried out with commercial AFM and LDV for Sader method and thermal tune. Tested probes allowed to compare the obtained spring constant values in range of two orders of magnitude $(0.36$ to $36 \mathrm{~N} / \mathrm{m})$. For the soft cantilevers, obtained results agreement was at the level of few percent. On the other hand for the stiff probe (100 $\mu \mathrm{m}$ in length), the differences between obtained values were significantly bigger. As the major reasons, Ohler listed: insufficient sensitivity of AFM optical detection system in case of thermal tune method, as the oscillation amplitude of the cantilever reaches approximately $12 \mathrm{~nm}$, and the $L / w$ ratio being below the limitation of the Sader method.

Also Gates and Pratt used the Doppler vibrometry in order to measure the oscillations of the cantilever in terms of improving the thermal noise method [151]. Due to unique sensitivity, such an approach allows to analyze both: longitudinal and torsional oscillations of the cantilever. Basing on the Euler-Bernoulli model and utilization of electrostatic balance technique providing SI standards traceability, complete calibration procedure can be performed.

Another attempt of the comparison between various calibration techniques was presented by Kim et al. [152]. In this experiment, following methods were utilized: the nano force calibrator (NFC), Euler-Bernoulli formula, thermal noise method, Sader method and cantilever on cantilever technique. Commercial probes for contact $(0.9 \mathrm{~N} / \mathrm{m}$ nominal $)$ and semicontact ( $42 \mathrm{~N} / \mathrm{m}$ nominal) were calibrated. Obtained results revealed good agreement between NFC, COC and thermal noise method for soft probe. Sader method delivered significantly lower spring constant values, the outcome of theoretical calculations on the other hand, was significantly higher. For the stiff probe, COC and NFC showed good correlation, theoretical calculations delivered significantly higher values, and for Sader method, the spring constant values were lower.

In order to verify the accuracy of certain measurement devices, the round robin procedures are carried out as standard metrological procedure. Due to advanced development level of the determination of the spring constant of the AFM's such a practice was also introduced. The results presented by te Riet et al. [120]. Various probes (rectangular and V-shaped) were used in order to perform the procedure. Thermal noise an Sader method were utilized in the comparison. 10 AFM systems were involved: 2 custom built (including on base of Asylum MFP-3D, JPK NanoWizard I, Agilent 5500 and 6 systems basing on controllers Multimode Nanoscope IIIa, IV, IVa and V. Obtained results showed good correlation of obtained results, however some issues in terms of systematic errors were revealed for few setups. It was also concluded, that Sader method allowed to obtain uncertainty $3 \%$ to $7 \%$ for single AFM and average value respectively. On the other hand, for thermal noise method, the uncertainty reached $6 \%$ to $15 \%$ likewise.

\section{Summary and Outlook}

In this work the most popular and interesting methods of the AFM probes spring constant determination were presented. In addition, practical utilization examples were also shown. The increasing demand of the quantitative AFM forces measurements, caused development of many techniques offering various levels of complexity and accuracy. Although 
easy, cheap, quick, reliable and high accuracy calibration methods are desired, also sophisticated and requiring advanced equipment ones were also implemented and described in many papers. The diversity of approaches provides an insight into the progress of understanding the phenomena of various aspects of atomic forces microscopy, as well as the rapid technology development. During last three decades, a plenty of solutions have been implemented and improved, and some of them become worldwide popular and commonly used. One has to keep in mind, that despite the fact, that some of the methods are easily accessible in commercial AFM setups and require a very little effort to perform, their application range is limited. In addition, for each technique a certain steps must be taken in order to reduce the error sources as much as possible. It was also shown, that the utilization of few methods for selected group of probes, providing correlation of the outcomes, allows to obtain an insight into the potential weaknesses of specific procedures performed in the lab.

\section{Acknowledgments}

This work was performed within statute funds of Electrotechnical Institute. The kind support of authors who provided the figures is acknowledged.

\section{References}

[1] G. Binnig, C. F. Quate, and C. Gerber, "Atomic force microscope," Phys. Rev. Lett., vol. 56, pp. 930-933, 1986.

[2] S. Morita, Roadmap of Scanning Probe Microscopy. Springer Berlin Heidelberg, 2007.

[3] H. K. Wickramasinghe, "Progress in scanning probe microscopy," Acta Mater, vol. 48, no. 1, pp. 347-358, Jan. 2000 .

[4] B. Bhushan, Springer Handbook of Nanotechnology. Berlin, Heidelberg: Springer Berlin Heidelberg, 2010.

[5] S. P. Jarvis and S. Yamamoto, "Tip-surface interactions studied using a force controlled atomic force microscope in ultrahigh vacuum," Physics (College. Park. Md)., vol. 70, no. April, pp. 2238-2240, 1997.

[6] E. Radlein, R. Ambos, and G. H. Frischat, "Atomic force microscopy of coated glasses," J. Anal. Chem., vol. 353, pp. 413-418, 1995.

[7] S. Lesko, E. Lesniewska, A. Nonat, J. C. Mutin, and J. P. Goudonnet, "Investigation by atomic force microscopy of forces at the origin of cement cohesion.," Ultramicroscopy, vol. 86, no. 1-2, pp. 11-21, Jan. 2001

[8] R. Giles, J. P. Cleveland, S. Manne, P. K. Hansma, B. Drake, P. Maivald, C. Boles, J. Gurley, and V. Elings, "Noncontact force microscopy in liquids," Appl. Phys. Lett., vol. 63, no. 5, p. 617, 1993.

[9] A. Altes, R. Heiderhoff, and L. J. Balk, "Quantitative dynamic near-field microscopy of thermal conductivity," J. Phys. D. Appl. Phys., vol. 37, no. 6, pp. 952-963, Mar. 2004.
[10] G. Bernhard, M. Fiege, A. Altes, and R. Heiderhoff, "Quantitative thermal conductivity measurements with nanometre resolution," J. Phys. D Appl. Phys., vol. 32, pp. L13-L17, 1999.

[11] V. V. Zavyalov, J. S. McMurray, and C. C. Williams, "Scanning capacitance microscope methodology for quantitative analysis of $\mathrm{p}$ - n junctions," J. Appl. Phys., vol. 85, no. 11 , pp. $7774-7783,1999$.

[12] B.-F. Ju, Y. Ju, and M. Saka, "Quantitative measurement of submicrometre electrical conductivity," J. Phys. D. Appl. Phys., vol. 40, no. 23, pp. 7467-7470, Dec. 2007.

[13] U. Weißker, S. Philippi, A. Leonhardt, P. Banerjee, A. Reed, F. Wolny, Y. Obukhov, T. M, G. Xiang, R. Adur, I. Lee, A. J. Hauser, F. Y. Yang, D. V Pelekhov, B. Büchner, and P. C. Hammel, "Ultramicroscopy Quantitative magnetic force microscopy on permalloy dots using an iron filled carbon nanotube probe," Ultramicroscopy, vol. 2, pp. 1-6, 2011.

[14] S. Jeffery, A. Oral, and J. B. Pethica, "Quantitative electrostatic force measurement in AFM," Appl. Surf. Sci., pp. 280-284, 2000.

[15] A. Gigler, C. Gnahm, O. Marti, T. Schimmel, and S. Walheim, "Towards quantitative materials characterization with Digital Pulsed Force Mode imaging," J. Phys. Conf. Ser., vol. 61, pp. 346-351, Mar. 2007.

[16] A. Cuenat, A. Muñiz-Piniella, M. Muñoz-Rojo, W. C. Tsoi, and C. E. Murphy, "Quantitative nanoscale surface voltage measurement on organic semiconductor blends.," Nanotechnology, vol. 23, no. 4, p. 045703, Feb. 2012.

[17] B. Cappella and G. Dietler, "Force-distance curves by atomic force microscopy," Surf. Sci. Rep., vol. 34, no. 1-3, pp. 1-104, 1999.

[18] M. Brogly, H. Awada, and O. Noel, "4 Contact Atomic Force Microscopy: A Powerful Tool in Adhesion Science," in Applied Scanning Probe Methods XI, B. Bhushan and H. Fuchs, Eds. Springer-Verlag Berlin Heidelberg, 2009, pp. 73-95.

[19] A. Ptak, H. Gojzewski, M. Kappl, and H.-J. Butt, "Influence of humidity on the nanoadhesion between a hydrophobic and a hydrophilic surface," Chem. Phys. Lett., vol. 503, no. 1-3, pp. 66-70, Feb. 2011.

[20] C. Gu, C. Ray, S. Guo, and B. B. Akhremitchev, "Single-Molecule Force Spectroscopy Measurements of Interactions between C 60 Fullerene Molecules," pp. 12898-12905, 2007.

[21] C. C. Wu, H. W. Su, C. C. Lee, M. J. Tang, and F. C. Su, "Quantitative measurement of changes in adhesion force involving focal adhesion kinase during cell attachment, spread, and migration," Biochem. Biophys. Res. Commun., vol. 329, no. 1, pp. 256-265, 2005.

[22] D. Craig, A. Krammer, K. Schulten, and V. Vogel, "Comparison of the early stages of forced unfolding for fibronectin type III modules.," Proc. Natl. Acad. Sci. U. S. A., vol. 98, no. 10, pp. 5590-5, May 2001.

[23] A. Sikora and A. Iwan, "AFM study of the mechanical wear phenomena of the polyazomethine with thiophene rings: Tapping mode, phase imaging mode and force spectroscopy," High Performance Polymers, vol. 24, no. 3. pp. 218-228, 2012. 
[24] F. Spectroscopy, W. Shi, Y. Zhang, C. Liu, Z. Wang, and X. Zhang, "Interaction between Dendrons Directly Studied by Single-Molecule," Synthesis (Stuttg)., no. 29, pp. 1318-1323, 2008.

[25] J. J. Roa, G. Oncins, F. T. Dias, V. N. Vieira, J. Schaf, and M. Segarra, "AFM as an alternative for Young's modulus determination in ceramic materials in elastic deformation regime," Phys. C Supercond. its Appl., vol. 471, no. 17-18, pp. 544-548, 2011.

[26] E. Canetta and A. K. Adya, "Atomic force microscopic investigation of commercial pressure sensitive adhesives for forensic analysis.," Forensic Sci. Int., vol. 210, no. 1-3, pp. 16-25, Jul. 2011.

[27] S. Strasser, A. Zink, G. Kada, P. Hinterdorfer, O. Peschel, W. M Heckl, A. G. Nerlich, and S. Thalhammer, "Age determination of blood spots in forensic medicine by force spectroscopy.," Forensic Sci. Int., vol. 170, no. 1, pp. 8-14, Jul. 2007.

[28] B. Bhushan, "Nanotribology and nanomechanics," Wear, vol. 259, no. 7-12, pp. 1507-1531, Jul. 2005.

[29] D. B. Serrell, J. Law, A. J. Slifka, R. L. Mahajan, and D. S. Finch, "A uniaxial bioMEMS device for imaging single cell response during quantitative force-displacement measurements," Biomed. Microdevices, vol. 10, no. 6, pp. 883-889, 2008.

[30] "NT-MDT company webpage/ Etalon probes information," 2009. [Online].

Available: http://www.ntmdt.com/press-releases/view/next-generation-of -afm-probes-etalon-series-best-price-best-quality. [Accessed: 07-Jul-2015].

[31] F. J. Giessibl, "Atomic resolution on $\mathrm{Si}(111)-(7 \times 7)$ by noncontact atomic force microscopy with a force sensor based on a quartz tuning fork," Appl. Phys. Lett., vol. 76, no. 11, p. 1470,2000

[32] F. J. Giessibl, "Subatomic Features on the Silicon (111)-(7x7) Surface Observed by Atomic Force Microscopy," Science, vol. 289, no. 5478. pp. 422-425, 2000.

[33] “Micro Star Technologies company webpage," 2012. [Online]. Available: http://www.microstartech.com/. [Accessed: 07-Jul-2015].

[34] G. Meyer and N. M. Amer, "Novel optical approach to atomic force microscopy," Appl. Phys. Lett., vol. 53, no. 12, pp. 1045-1047, 1988.

[35] H. Xie, J. Vitard, D. S. Haliyo, and S. Régnier, "Enhanced accuracy of force application for AFM nanomanipulation using nonlinear calibration of optical levers," IEEE Sens. J., vol. 8, no. 8, pp. 1478-1485, 2008.

[36] K. Nieradka, G. Jóźwiak, D. Kopiec, P. Grabiec, P. Janus, A. Sierakowski, and T. Gotszalk, "A method for linearization of split photodiode position detectors response," Procedia Eng., vol. 25, pp. 358-361, 2011.

[37] K. Nieradka, G. Małozięć, D. Kopiec, P. Grabiec, P. Janus, a Sierakowski, and T. Gotszalk, "Expanded beam deflection method for simultaneous measurement of displacement and vibrations of multiple microcantilevers.," Rev. Sci. Instrum., vol. 82 , no. 10 , p. 105112 , Oct. 2011.

[38] D. Georgakaki, S. Mitridis, A. A. Sapalidis, E. Mathioulakis, and H. M. Polatoglou, "Calibration of tapping AFM cantilevers and uncertainty estimation: Comparison between different methods," Meas. J. Int. Meas. Confed., vol. 46, no. 10, pp. 4274-4281, 2013.

[39] J. E. Sader, I. Larson, P. Mulvaney, and L. R. White, "Method for the calibration of atomic force microscope cantilevers," Rev. Sci. Instrum., vol. 66, no. 7, pp. 3789-3798, 1995.

[40] C. T. Gibson, B. L. Weeks, C. Abell, T. Rayment, and S. Myhra, "Calibration of AFM cantilever spring constants," Ultramicroscopy, vol. 97, no. 1-4, pp. 113-118, 2003.

[41] “AIST-NT webpage." [Online]. Available: http://www.aist-nt.com/products/smartspmtm-1000. [Accessed: 07-Jul-2015].

[42] S. Rode, R. Stark, J. Lübbe, L. Tröger, J. Schütte, K. Umeda, K. Kobayashi, H. Yamada, and A. Kühnle, "Modification of a commercial atomic force microscopy for low-noise, high-resolution frequency-modulation imaging in liquid environment," Rev. Sci. Instrum., vol. 82, no. 7, p. 073703, 2011.

[43] D. Rugar, H. J. J. Mamin, and P. Guethner, "Improved fiber-optic interferometer for atomic force microscopy," Appl. Phys. Lett., vol. 55, no. 25, pp. 2588-2590, 1989.

[44] J. Chu, T. Itoh, C. Lee, T. Suga, and K. Watanabe, "Novel high vacuum scanning force microscope using a piezoelectric cantilever and the phase detection method," J. Vac. Sci. Technol. B Microelectron. Nanom. Struct., vol. 15, no. 4, pp. 1551-1555, 1997.

[45] T. Gotszalk, P. Grabiec, E. Tomerov, and I. W. Rangelow, "Thermally driven micromechanical beam with piezoresistive deflection readout," Microelectron. Eng., vol. 68, pp. 550-556, 2003.

[46] E. M. Chow, G. G. Yaralioglu, C. F. Quate, and T. W. Kenny, "Characterization of a two-dimensional cantilever array with through-wafer electrical interconnects," Appl. Phys. Lett., vol. 80, no. 4, pp. 664-666, 2002.

[47] I. W. Rangelow, T. Ivanov, K. Ivanova, B. E. Volland, P. Grabiec, Y. Sarov, A. Persaud, T. Gotszalk, P. Zawierucha, M. Zielony, D. Dontzov, B. Schmidt, M. Zier, N. Nikolov, I. Kostic, W. Engl, T. Sulzbach, J. Mielczarski, S. Kolb, D. P. Latimier, R. Pedreau, V. Djakov, S. E. Huq, K. Edinger, O. Fortagne, A. Almansa, and H. O. Blom, "Piezoresistive and self-actuated 128-cantilever arrays for nanotechnology applications," Microelectron. Eng., vol. 84, no. 5-8, pp. 1260-1264, May 2007.

[48] A. Schneider, R. H. Ibbotson, R. J. Dunn, V. Djakov, E. Huq, and T. Gotszalk, "Parallel Imaging with Arrays of SU-8 Microcantilevers," no. September, 2010.

[49] J. M. Łysko, P. Dumania, P. Janus, M. Grodner, H. Kłos, K. Skwara, and Grab, "Nanosensitive Silicon Microprobes for Mechanical Detection and Measurements," Mater. Sci. Appl., vol. 02 , no. 06, pp. 582-591, 2011.

[50] D. Kopiec, P. Pałetko, K. Nieradka, W. Majstrzyk, P. Kunicki, A. Sierakowski, G. Jóźwiak, and T. Gotszalk, "Closed-loop surface stress compensation with an electromagnetically actuated microcantilever," Sensors Actuators B Chem., vol. 213, pp. 566-573, 2015.

[51] T. Göddenhenrich, H. Lemke, U. Hartmann, and C. Heiden, "Force microscope with capacitive displacement detection," $J$. Vac. Sci. Technol. A, vol. 8, pp. 383-387, 1990. 
[52] G. Binnig, C. Gerber, E. Stoll, T. R. Albrecht, and C. F. Quate, "Atomic Resolution with Atomic Force Microscope," Europhys. Lett., vol. 3, no. 12, pp. 1281-1286, Jun. 1987.

[53] R. Leach, J. Haycocks, K. Jackson, A. Lewis, S. Oldfield, and A. Yacoot, "Advances in traceable nanometrology at the National Physical Laboratory," Nanotechnology, vol. 12, p. R1, 2001.

[54] J. Hrabina, J. Lazar, P. Klapetek, and O. Číp, "Multidimensional interferometric tool for the local probe microscopy nanometrology," Meas. Sci. Technol., vol. 22, no. 9 , p. 94030,2011 .

[55] M. Xu, T. Dziomba, G. Dai, and L. Koenders, "Self-calibration of scanning probe microscope: mapping the errors of the instrument," Meas. Sci. Technol., vol. 19, no. 2, p. 025105, Feb. 2008.

[56] S. Strube, G. Molnar, and H.-U. Danzebrink, "Compact field programmable gate array (FPGA)-based multi-axial interferometer for simultaneous tilt and distance measurement in the sub-nanometre range," Meas. Sci. Technol., vol. 22, no. 9, p. 94026 , Sep. 2011.

[57] M. Zielony and T. Gotszalk, "Digital setting of scanning field in scanning probe microscopy," Prz. Elektrotechniczny, vol. 86, no. 11 A, pp. $180-183,2010$.

[58] D. Nečas and P. Klapetek, "Gwyddion: an open-source software for SPM data analysis," Cent. Eur. J. Phys., vol. 10, no. 1, pp. 181-188, Nov. 2011.

[59] "SPIP online manual." [Online]. Available: http://www.imagemet.com/WebHelp6/Default.htm. [Accessed: 07-Jul-2015].

[60] I. Horcas, R. Fernández, J. M. Gómez-Rodríguez, J. Colchero, J. Gómez-Herrero, and a M. Baro, "WSXM: a software for scanning probe microscopy and a tool for nanotechnology.," Rev. Sci. Instrum., vol. 78, no. 1, p. 13705, Jan. 2007.

[61] J. M. Skwierczynski, G. Maloziec, D. Kopiec, K. Nieradka, J. Radojewski, and T. P. Gotzszalk, "Radio frequency modulation of semiconductor laser as an improvement method of noise performance of scanning probe microscopy position sensitive detectors," Opt. Appl., vol. 41, no. 2, pp. 323-331, 2011.

[62] A. Ptak, M. Kappl, and H. J. Butt, "Modified atomic force microscope for high-rate dynamic force spectroscopy," Appl. Phys. Lett., vol. 88, no. 26, p. 263109, 2006.

[63] W. F. Heinz and J. H. Hoh, "Spatially resolved force spectroscopy of biological surfaces using the atomic force microscope.," Trends Biotechnol., vol. 17, no. 4, pp. 143-150, 1999.

[64] C. Reynaud, F. Sommer, C. Quet, N. El Bounia, T. M. Duc, and N. El Bounia, "Quantitative determination of Young's modulus on a biphase polymer system using atomic force microscopy," Surf. Interface Anal., vol. 189, no. 30, pp. 185-189, Aug. 2000.

[65] P. J. de Pablo, J. Colchero, J. Gómez-Herrero, A. M. Baró, P. J. De Pablo, and J. Gomez-Herrero, "Jumping mode scanning force microscopy," Appl. Phys. Lett., vol. 73, no. 22, pp. 3300-3302, 1998.

[66] F. Moreno-Herrero, J. Colchero, J. Gómez-Herrero, A. M. Baró and J. Ávila, "Jumping mode atomic force microscopy obtains reproducible images of Alzheimer paired helical filaments in liquids," Eur. Polym. J., vol. 40, no. 5, pp. 927-932, 2004.
[67] E. Weilandt, S. Hild, O. Marti, and A. Rosa-Zeiser, "The simultaneous measurement of elastic, electrostatic and adhesive properties by scanning force microscopy: pulsed-force mode operation," Meas. Sci. Technol., vol. 8, pp. 1333-1338, 1997.

[68] J. Legleiter, M. Park, B. Cusick, and T. Kowalewski, "Scanning probe acceleration microscopy (SPAM) in fluids: mapping mechanical properties of surfaces at the nanoscale.," Proc. Natl. Acad. Sci. U. S. A., vol. 103, no. 13, pp. 4813-8, Mar. 2006.

[69] D. M. Panaitescu, A. N. Frone, M. Ghiurea, and I. Chiulan, "Influence of storage conditions on starch/PVA films containing cellulose nanofibers," Ind. Crops Prod., vol. 70, pp. $170-177,2015$.

[70] L. Chopinet, C. Formosa, M. P. Rols, R. E. Duval, and E. Dague, "Imaging living cells surface and quantifying its properties at high resolution using $\mathrm{AFM}$ in $\mathrm{QI}^{\mathrm{TM}}$ mode.," Micron, vol. 48, pp. 26-33, 2013.

[71] O. Sahin, "Harnessing bifurcations in tapping-mode atomic force microscopy to calibrate time-varying tip-sample force measurements," Rev. Sci. Instrum., vol. 78, no. 103707, pp. 1-4, 2007.

[72] O. Sahin, S. Magonov, C. Su, C. F. Quate, and O. Solgaard, “An atomic force microscope tip designed to measure time-varying nanomechanical forces," Nat. Nanotechnol., vol. 2, no. 8, pp. 507-514, 2007.

[73] A. Sikora and L. Bednarz, "Mapping of the surface's mechanical properties due to analysis of torsional cantilever bending in dynamic force microcopy," in Scanning Probe Acoustic Techniques, F. Marinello, D. Passeri, and E. Savio, Eds. Springer Berlin Heidelberg, 2012, p. w druku.

[74] A. Sikora, M. Woszczyna, M. Friedemann, F. J. Ahlers, and M. Kalbac, "AFM diagnostics of graphene-based quantum Hall devices.," Micron, vol. 43, no. 2-3, pp. 479-86, Feb. 2012.

[75] M. G. Reitsma, R. S. Gates, and R. F. Cook, "Torsional spring constant measurement of a T-shaped atomic force microscope cantilever Materials Science and Engineering Laboratory," Exp. Mech., 2009.

[76] J. A. Greenwood and K. L. Johnson, "Mechanics of adhesion of viscoelastic solids," Philos. Mag. A Phys. Condens. Matter, Struct. Defects Mech. Prop., vol. 43, no. 3, pp. 697-711, 1981.

[77] B. V Derjaguin, V. M. Muller, and Y. U. P. Toporov, "Effect of contact deformations on the adhesion of particles," J. Colloid Interface Sci., vol. 53, pp. 314-326, 1975.

[78] J. K. Kim, D. E. Lee, W. Il Lee, and K. Y. Suh, "Measurement of pull-off force on imprinted nanopatterns in an inert liquid.," Nanotechnology, vol. 21, no. 29, p. 295306, Jul. 2010.

[79] D. Xu and K. M. Liechti, "On the modified Tabor parameter for the JKR - DMT transition in the presence of a liquid meniscus," vol. 315, pp. 772-785, 2007.

[80] E. Boer-Duchemin, E. Tranvouez, and G. Dujardin, "The interaction of an atomic force microscope tip with a nano-object: a model for determining the lateral force.," Nanotechnology, vol. 21, no. 45, p. 455704, Nov. 2010.

[81] J. Drelich, G. W. Tormoen, and E. R. Beach, "Determination of solid surface tension from particle-substrate pull-off forces measured with the atomic force microscope.," J. Colloid Interface Sci., vol. 280, no. 2, pp. 484-97, Dec. 2004. 
[82] C. Clifford and M. P. Seah, "Improved methods and uncertainty analysis in the calibration of the spring constant of an atomic force microscope cantilever using static experimental methods," Meas. Sci. Technol., vol. 20, no. 12, p. 125501, Dec. 2009.

[83] G. A. Matei, E. J. Thoreson, J. R. Pratt, D. Newell, and N. A. Burnham, "Precision and accuracy of thermal calibration of atomic force microscopy cantilevers," Rev. Sci. Instrum., vol. 77 , no. 8 , p. 083703,2006

[84] C. T. Gibson, D. Alastair Smith, and C. J. Roberts, "Calibration of silicon atomic force microscope cantilevers.," Nanotechnology, vol. 16, no. 2, pp. 234-238, 2005.

[85] "ISO 27911:2011, Surface chemical analysis -- Scanning-probe microscopy -- Definition and calibration of the lateral resolution of a near-field optical microscope."

[86] “ISO 11039:2012, Surface chemical analysis -- Scanning-probe microscopy -- Measurement of drift rate.”

[87] "ISO 11952:2014, Surface chemical analysis -- Scanning-probe microscopy -- Determination of geometric quantities using SPM: Calibration of measuring systems."

[88] "ISO 13095:2014, Surface Chemical Analysis -- Atomic force microscopy -- Procedure for in situ characterization of AFM probe shank profile used for nanostructure measurement."

[89] “ISO/DIS 11775, Surface chemical analysis -- Scanning-probe microscopy -- Determination of cantilever normal spring constants."

[90] T. R. Albrecht, S. Akamine, T. E. Carver, C. F. Quate, and E. L. Ginzton, "Microfabrication of cantilever styli for the atomic force microscope," J. Vac. Sci. Technol. A Vacuum, Surfaces, Film., vol. 94305, no. January, pp. 3386-3396, 1990.

[91] H.-J. Butt, P. Siedle, K. Seifert, K. Fendler, T. Seeger, E. Bamberg, A. L. Weisenhorn, K. Goldie, and A. Engel, "Scan Speed Limit In Atomic Force Microscopy," J. Microsc., vol. 169, no. 1, pp. 75-84, Jan. 1993.

[92] J. E. Sader, "Parallel beam approximation for V-shaped atomic force microscope cantilevers," Rev. Sci. Instrum., vol. 66, no. 9, pp. 4583-4587, 1995.

[93] J. E. Sader, J. W. M. Chon, and P. Mulvaney, "Calibration of rectangular atomic force microscope cantilevers," Rev. Sci. Instrum., vol. 70, no. 10, pp. 3967-3969, 1999.

[94] J. W. M. Chon, P. Mulvaney, and J. E. Sader, "Experimental validation of theoretical models for the frequency response of atomic force microscope cantilever beams immersed in fluids," J. Appl. Phys., vol. 87, no. 8, pp. 3978-3988, 2000.

[95] H. Frentrup and M. S. Allen, "Error in dynamic spring constant calibration of atomic force microscope probes due to nonuniform cantilevers.," Nanotechnology, vol. 22, no. 29, p. 295703, Jul. 2011.

[96] J. Bowen, D. Cheneler, D. Walliman, S. G. Arkless, Z. Zhang, M. C. L. Ward, and M. J. Adams, "On the calibration of rectangular atomic force microscope cantilevers modified by particle attachment and lamination," Meas. Sci. Technol., vol. 21, no. 11, p. 115106, Nov. 2010.

[97] J. M. Neumeister and W. A. Ducker, "Lateral, normal, and longitudinal spring constants of atomic force microscopy cantilevers," Rev. Sci. Instrum., vol. 65, no. 8, pp. 2527-2531, 1994.
[98] C. A. Clifford and M. P. Seah, "The determination of atomic force microscope cantilever spring constants via dimensional methods for nanomechanical analysis," Nanotechnology, vol. 16, no. 9. pp. 1666-1680, 2005.

[99] B. Y. Chen, M. K. Yeh, and N. H. Tai, "Accuracy of the spring constant of atomic force microscopy cantilevers by finite element method," Anal. Chem., vol. 79, no. 4, pp. 1333-1338, 2007.

[100] J. P. Cleveland, S. Manne, D. Bocek, and P. K. Hansma, "A nondestructive method for determining the spring constant of cantilevers for scanning force microscopy A nondestructive method for determining the spring constant of cantilevers for scanning force microscopy," vol. 403, no. 1993, 2012.

[101] T. Gotszalk, P. B. Grabiec, and I. W. Rangelow, "A novel piezoresistive microprobe for atomic and lateral force microscopy," Sensors Actuators A Phys., vol. 123-124, pp. 370-378, Sep. 2005.

[102] F. Loizeau, T. Akiyama, S. Gautsch, A. Meister, P. Vettiger, and N. De Rooij, "2D cantilever array with fixed geometries and varying spring constants for life science applications," Procedia Eng., vol. 25, pp. 669-672, 2011.

[103] J. L. Hutter and J. Bechhoefer, "Calibration of atomic-force microscope tips," Rev. Sci. Instrum., vol. 64, no. 7, pp. 1868-1873, 1993

[104] M. Jaschke, "Calculation of thermal noise," Energy, vol. 6, pp. $1-7,1995$.

[105] R. Levy and M. Maaloum, "Measuring the spring constant of atomic force microscope cantilevers: thermal fluctuations and other methods," Nanotechnology, vol. 13, p. 33, 2002.

[106] M. S. Allen, H. Sumali, and P. C. Penegor, "Effect of Tip Mass on Atomic Force Microscope Calibration by Thermal Method," in Conference: 2009 IMAC-XXVII: Conference \& Exposition on Structural Dynamics, 2009, pp. 1-9.

[107] R. Proksch, T. E. Schäffer, J. P. Cleveland, R. C. Callahan, and M. B. Viani, "Finite optical spot size and position corrections in thermal spring constant calibration," Nanotechnology, vol. 15, no. 9. pp. 1344-1350, 2004.

[108] “Online ODB sensitivity calculator,” 2015. [Online]. Available: http://www.bioforce.uni-tuebingen.de/aspx/OBDcalc/OBDcalc. aspx.

[109] L. O. Heim, T. S. Rodrigues, and E. Bonaccurso, "Direct thermal noise calibration of colloidal probe cantilevers," Colloids Surfaces A Physicochem. Eng. Asp., vol. 443, pp. 377-383, 2014.

[110] M. Boudaoud, Y. Haddab, Y. Le Gorrec, and P. Lutz, "Study of thermal and acoustic noise interferences in low stiffness atomic force microscope cantilevers and characterization of their dynamic properties," Review of Scientific Instruments, vol. 83, no. 1.2012.

[111] S. F. Nørrelykke and H. Flyvbjerg, "Power spectrum analysis with least-squares fitting: Amplitude bias and its elimination, with application to optical tweezers and atomic force microscope cantilevers," Rev. Sci. Instrum., vol. 81, no. 7, pp. $1-16,2010$.

[112] E. Bonaccurso and H. J. Butt, "Microdrops on atomic force microscope cantilevers: Evaporation of water and spring constant calibration," J. Phys. Chem. B, vol. 109, no. 1, pp. 253-263, 2005. 
[113] J. P. Cleveland, S. Manne, D. Bocek, and P. K. Hansma, “A nondestructive method for determining the spring constant of cantilevers for scanning force microscopy," Rev. Sci. Instrum. vol. 64 , no. 2 , p. $403,1993$.

[114] Y. M. Tseytlin, "Kinetostatic model of spring constant ratios for an AFM cantilever with end extended mass," Ultramicroscopy, vol. 110, no. 2, pp. 126-129, 2010.

[115] G. Jóźwiak, D. Kopiec, P. Zawierucha, T. Gotszalk, P. Janus, P. Grabiec, and I. W. Rangelow, "The spring constant calibration of the piezoresistive cantilever based biosensor," Sensors Actuators, B Chem., vol. 170, pp. 201-206, 2012.

[116] D. Kopiec, "PhD thesis: The properties and utilization of the micro- and nanomechanical tools for nanoforces measurements.," Wrocław University of Technology, 2015.

[117] M. Woszczyna, P. Zawierucha, M. Świątkowski, T. Gotszalk, P. Grabiec, N. Nikolov, J. Mielczarski, E. Mielczarska, N. Glezos, T. Ivanow, K. Ivanowa, Y. Sarov, and I. W. Rangelow, "Quantitative force and mass measurements using the cantilever with integrated actuator and deflection detector," Microelectron. Eng., vol. 86, no. 4-6, pp. 1043-1045, Apr. 2009.

[118] J. Singh and J. E. Whitten, "Forces between Polymer Surfaces and Self-Assembled Monolayers," J. Macromol. Sci. Part A, vol. 45, no. 11, pp. 884-891, Sep. 2008.

[119] J. Melcher, C. Carrasco, X. Xu, J. L. Carrascosa, J Gómez-Herrero, P. José de Pablo, A. Raman, J. Melchera, C. Carrascob, X. Xua, J. L. Carrascosad, J. Gomez-Herrerob, P. J. de Pablob, and A. Ramana, "Origins of phase contrast in the atomic force microscope in liquids.," Proc. Natl. Acad. Sci. U. S. A., vol. 106, no. 33, pp. 13655-13660, Aug. 2009.

[120] J. te Riet, A. J. Katan, C. Rankl, S. W. Stahl, A. M. van Buul, I. Y. Phang, A. Gomez-Casado, P. Schön, J. W. Gerritsen, A. Cambi, A. E. Rowan, G. J. Vancso, P. Jonkheijm, J. Huskens, T. H. Oosterkamp, H. Gaub, P. Hinterdorfer, C. G. Figdor, and S. Speller, "Interlaboratory round robin on cantilever calibration for AFM force spectroscopy," Ultramicroscopy, vol. 111, no. 12, pp. 1659-1669, 2011.

[121] B. Ohler, "Cantilever spring constant calibration using laser Doppler vibrometry," Rev. Sci. Instrum., vol. 78, no. 6, pp. 10-15, 2007.

[122] D.-A. Mendels, M. Lowe, A. Cuenat, M. G. Cain, E. Vallejo, D. Ellis, and F. Mendels, "Dynamic properties of AFM cantilevers and the calibration of their spring constants," $J$. Micromechanics Microengineering, vol. 16, no. 8, pp. 1720-1733, 2006.

[123] J. D. Holbery, V. L. Eden, M. Sarikaya, and R. M. Fisher, "Experimental determination of scanning probe microscope cantilever spring constants utilizing a nanoindentation apparatus," Rev. Sci. Instrum., vol. 71, no. 10, pp. 3769-3776, 2000 .

[124] J. D. Holbery and V. L. Eden, “A comparison of scanning microscopy cantilever force constants determined using a nanoindentation testing apparatus," J. Micromechanics, vol. 10, no. 2000, pp. 85-92, 2000 .

[125] M.-S. Kim, J.-H. Choi, Y.-K. Park, and J.-H. Kim, "Atomic force microscope cantilever calibration device for quantified force metrology at micro- or nano-scale regime: the nano force calibrator (NFC)," Metrologia, vol. 43, no. 5, pp. 389-395, 2006.
[126] M. S. Kim, I. M. Choi, Y. K. Park, and D. I. Kang, "Atomic force microscope probe calibration by use of a commercial precision balance," Meas. J. Int. Meas. Confed., vol. 40, no. 7-8, pp. 756-760, 2007.

[127] C. T. Gibson, G. S. Watson, and S. Myhra, "Determination of the spring constants of probes for force microscopy/spectroscopy," Nanotechnology, vol. 7, no. 3. pp. 259-262, 1999.

[128] R. S. Gates and J. R. Pratt, "Prototype cantilevers for SI-traceable nanonewton force calibration," Meas. Sci. Technol., vol. 17, no. 10, pp. 2852-2860, 2006.

[129] M. G. Reitsma and R. S. Gates, "A New High Precision Procedure for AFM Probe Spring Constant Measurement using a Microfabricated Calibrated Reference Cantilever Array (CRCA)," NSTI-Nanotech 2006, vol. 1, pp. 785-788, 2006.

[130] A. D. Slattery, A. J. Blanch, J. S. Quinton, and C. T. Gibson, "Accurate measurement of Atomic Force Microscope cantilever deflection excluding tip-surface contact with application to force calibration," Ultramicroscopy, vol. 131, pp. 46-55, 2013.

[131] G. A. Shaw, J. Kramar, and J. Pratt, "SI-traceable spring constant calibration of microfabricated cantilevers for small force measurement," Exp. Mech., vol. 47, no. 1, pp. 143-151, 2007.

[132] E. D. Langlois, G. A. Shaw, J. A. Kramar, J. R. Pratt, and D. C. Hurley, "Spring constant calibration of atomic force microscopy cantilevers with a piezosensor transfer standard," Rev. Sci. Instrum., vol. 78, no. 9, 2007.

[133] P. J. Cumpson, C. A. Clifford, and J. Hedley, "Quantitative analytical atomic force microscopy: a cantilever reference device for easy and accurate AFM spring-constant calibration," Meas. Sci. Technol., vol. 15, no. 7, pp. 1337-1346, Jul. 2004.

[134] C. J. Tourek and S. Sundararajan, "An alternative method to determining optical lever sensitivity in atomic force microscopy without tip-sample contact," Rev. Sci. Instrum., vol. 81, no. 7,2010

[135]P. J. Cumpson and J. Hedley, "Accurate analytical measurements in the atomic force microscope: a microfabricated spring constant standard potentially traceable to the SI." Nanotechnology, vol. 14, no. 12, pp. 1279-1288, 2003.

[136] P. J. P. J. Cumpson, P. Zhdan, and J. Hedley, "Calibration of AFM cantilever stiffness: A microfabricated array of reflective springs," Ultramicroscopy, vol. 100, no. 3-4, pp. 241-251, Aug. 2004.

[137] P. J. Cumpson, J. Hedley, C. A. Clifford, X. Chen, and S. Allen, "Microelectromechanical system device for calibration of atomic force microscope cantilever spring constants between 0.01 and 4 N/m," J. Vac. Sci. Technol. A Vacuum, Surfaces, Film., vol. 22, no. 4, p. 1444, 2004.

[138] R. Wagner, J. P. Killgore, R. C. Tung, A. Raman, and D. C. Hurley, "Vibrational shape tracking of atomic force microscopy cantilevers for improved sensitivity and accuracy of nanomechanical measurements," Nanotechnology, vol. 26, no. 4, p. 045701, 2015.

[139] M. Ekwińska, G. Ekwiński, and Z. Rymuza, "Calibration of normal force in atomic force microscope," in Recent Advantages in Mechatronics, Springer Berlin Heidelberg, 2007, pp. 505-510. 
[140] M. Ekwińska and Z. Rymuza, "Normal Force Calibration Method Used for Calibration of Atomic Force Microscope," vol. 116, pp. 78-81, 2009.

[141] "Nanoidea webpage." [Online]. Available: www.nanoidea.pl. [Accessed: 14-Jun-2013].

[142] A. Sikora, L. Bednarz, G. Ekwiński, and M. Ekwińska, "The determination of the spring constant of T-shaped cantilevers using calibration structures," Meas. Sci. Technol., vol. 25, no. 4, p. 044015, Apr. 2014.

[143] X. Li, D. Su, and Z. Zhang, "A novel technique of microforce sensing and loading," Sensors Actuators, A Phys., vol. 153, no. 1, pp. 13-23, 2009.

[144] B. Liu, Y. Yu, D. K. Yao, and J. Y. Shao, "A direct micropipette-based calibration method for atomic force microscope cantilevers," Rev. Sci. Instrum., vol. 80, no. 6, pp. $1-9,2009$.

[145] S. M. Notley, S. Biggs, and V. S. J. Craig, "Calibration of colloid probe cantilevers using the dynamic viscous response of a confined liquid," Rev. Sci. Instrum., vol. 74, no. 9, pp. 4026-4032, 2003.

[146] V. Kelley and R. Wishengrad, "PeakForce QNM User Guide 004-1036-000," 2011.
[147] C. T. Gibson, D. J. Johnson, C. Anderson, C. Abell, and T. Rayment, "Method to determine the spring constant of atomic force microscope cantilevers," Rev. Sci. Instrum., vol. 75, no. 2, pp. 565-567, 2004.

[148] G. B. Webber, G. W. Stevens, F. Grieser, R. R. Dagastine, and D. Y. C. Chan, "Variations in properties of atomic force microscope cantilevers fashioned from the same wafer.," Nanotechnology, vol. 19, no. 10, p. 105709, 2008.

[149] S. A. Edwards, W. A. Ducker, and J. E. Sader, "Influence of atomic force microscope cantilever tilt and induced torque on force measurements," J. Appl. Phys., vol. 103, no. 6, 2008.

[150] J. L. Hutter, "Comment on Tilt of Atomic Force Microscopy Cantilevers," Langmuir, vol. 21, pp. 2630-2632, 2005.

[151] R. S. Gates and J. R. Pratt, "Accurate and precise calibration of AFM cantilever spring constants using laser Doppler vibrometry," Nanotechnology, vol. 23, no. 37. p. 375702, 2012.

[152] M.-S. Kim, J.-H. Choi, J.-H. Kim, and Y.-K. Park, “Accurate determination of spring constant of atomic force microscope cantilevers and comparison with other methods," Measurement, vol. 43, no. 4, pp. 520-526, May 2010. 